\title{
Dual description of the superconducting phase transition
}

\author{
Michael Kiometzis, Hagen Kleinert, and Adriaan M.J. Schakel \\ Institut für Theoretische Physik \\ Freie Universität Berlin \\ Arnimallee 14 \\ 14195 Berlin
}

\begin{abstract}
The dual approach to the Ginzburg-Landau theory of a Bardeen-CooperSchrieffer superconductor is reviewed. The dual theory describes a grand canonical ensemble of fluctuating closed magnetic vortices, of arbitrary length and shape, which interact with a massive vector field representing the local magnetic induction. When the critical temperature is approached from below, the magnetic vortices proliferate. This is signaled by the disorder field, which describes the loop gas, developing a non-zero expectation value in the normal conducting phase. It thereby breaks a global U(1) symmetry. The ensuing Goldstone field is the magnetic scalar potential. The superconducting-tonormal phase transition is studied by applying renormalization group theory to the dual formulation. In the regime of a second-order transition, the critical exponents are given by those of a superfluid with a reversed temperature axis.
\end{abstract}

\section{INTRODUCTION}

In a recent letter [1] critical indices of the superconducting-to-normal phase transition were derived starting from a dual formulation of the Ginzburg-Landau theory. The purpose of the present article is to review this approach. The article, which is pedagogical in tone 
and attempts to be self-contained, highlights also some recent developments related to the symmetry aspects of the dual formulation which are important for understanding the phase transition.

The basic idea of the dual approach originates from three-dimensional $(3 D)$ lattice studies carried out more than 15 years ago [2 [5]. These studies were instigated by the success of the Berezinskii-Kosterlitz-Thouless (BKT) theory of the $2 D x y$ model developed in the early seventies to describe the phase transition in a superfluid film [6,7]. It was argued that the phase transition has its origin in the behavior of point-like vortices. In the ordered lowtemperature phase, pairs of vortices with opposite topological charge are tightly bound. Above the critical temperature, these pairs unbind and the liberated vortices disorder the system, turning the superfluid into a normal fluid.

The lattice studies of the pure 3D xy model [2] and its extension to a lattice superconductor [3,4] were aimed at obtaining an analogous description of the phase transitions in these models in terms of vortex loops. A detailed account of these matters as well as an extensive list of references to the literature can be found in the textbook by Kleinert [8].

Another development illuminating the dual approach to the Ginzburg-Landau model was initiated in Ref. [9]. The basic observation was that since a local gauge symmetry can never be broken [10, a local gauge description of a phase transition is not feasible. It was argued that the $3 D$ Ginzburg-Landau theory contains, in addition to the local gauge symmetry, another global U(1) symmetry. When considered in $2+1$ space-time dimensions with a Minkowski metric, this symmetry is generated by the magnetic flux operator. It was demonstrated that this symmetry is broken in the normal phase, while it is unbroken in the superconducting phase. An order parameter was given, and it was shown that the massless photon of the normal phase is the Goldstone particle associated with the broken flux symmetry.

To clarify the concept of duality it is expedient to remain for the moment in $2+1$ spacetime dimensions. There the theory is a quantum field theory possessing particle states which are created by field operators. This is to be contrasted with the space-time approach 
of Feynman [11] where worldlines of particles are the fundamental objects. The particle's mass is given by the tension of the worldline. Now, suppose that a quantum field theory contains point-like solitons, i.e., topologically stable, time-independent solutions of the field equations. When set into motion such a soliton traces out a non-trivial worldline in spacetime. An operator description of these excitations in terms of the original fields is non-local and quite difficult. The aim of the dual approach of the type we are considering is to describe them by a local field, called a disorder field [8]. The space-time description of the particle is thus converted into an equivalent field-theoretic one.

In the context of the (three dimensional Euclidean) Ginzburg-Landau theory the notion of a particle state is of course missing. The above picture has, however, a direct counterpart, viz., worldlines of the space-time approach correspond to vortices in the Euclidean theory. More precisely, in the setting of statistical (as opposed to quantum) field theory, a dual theory describes vortices by a disorder field. (In this discussion we restricted the notion of duality to line defects, thereby omitting dual theories of other topological defects such as domain walls and - in the context of quantum field theory - instantons.)

The advantage of the dual theory is that it allows for a simple description of the superconducting phase transition in the sense of Landau which is impossible in the original formulation, as we shall discuss. The order parameter of the transition is the field representing the magnetic vortices. This Landau theory, involving only a global symmetry and a genuine order parameter, can - as usual - be taken as a convenient starting point of a renormalization group analysis of the superconducting phase transition [1].

The layout of the paper is as follows. In the next section we illustrate the dual approach by considering the Ginzburg-Landau model in two space dimensions. This example gives us important clues towards the form of the dual formulation in $3 D$. In Sec. [1] we review the fact that a loop gas can be succinctly described near a critical point by a $|\psi|^{4}$ field theory. This equivalence plays a central role in the subsequent part of the paper. In Sec. पD we discuss the dual formulation of a $3 D$ lattice superconductor, and in Sec. $\nabla$ we review the formulation in the continuum. In Sec. VI we argue that the dual theory yields the 
standard description of the mixed state of a type-II superconductor. As a consistency check we consider in Sec. VII the dual formulation of the dual theory and demonstrate that the resulting theory is the original Ginzburg-Landau model. In Sec. VIII we discuss the gaugeinvariant superconducting order parameter. Finally, in Sec. $\mathbb{X} \mathbb{Z}$ we apply renormalization group theory to the dual theory to show that the superconducting phase transition is of second order. The critical exponents are shown to be in the universality class of the $x y$ model with the temperature axis reversed.

\section{DUAL MAP OF A $2 D$ SUPERCONDUCTOR}

Before discussing the theory of a superconductor in three space dimensions, it is instructive to first study the two-dimensional case. This exercise will exhibit the relevant variables in a dual description and show what in principle can be expected from such a description.

The Ginzburg-Landau model resulting from the Bardeen-Cooper-Schrieffer (BCS) theory of superconductivity is specified by the Hamiltonian

$$
H=\int d^{2} x\left[\frac{1}{2}(\nabla \times \mathbf{A})^{2}+|(\nabla-2 i e \mathbf{A}) \phi|^{2}+m_{\phi}^{2}|\phi|^{2}+\lambda|\phi|^{4}\right]
$$

with $\mathbf{A}$ the electromagnetic gauge potential. We adopt the standard notation and denote by $\nabla \times \mathbf{A}=\epsilon_{i j} \partial_{i} A_{j}$ the local induction. The symbol $B$ is reserved for the spatial average of the local field, and will be referred to as the magnetic induction, or flux density. These quantities have only one component in two space dimensions, pointing in the missing third direction. The coupling $2 e$ accounts for the double charge of Cooper pairs. The complex scalar field $\phi$ is the superconducting order field with a mass parameter $m_{\phi}$ and a self-coupling $\lambda$. In the superconducting phase of the model, $m_{\phi}^{2}<0$.

A useful limit of the theory is the so-called London limit where $m_{\phi}^{2} \rightarrow-\infty$ and $\phi$ can be written as $\phi(x)=\exp [i \theta(x)] w / \sqrt{2}$, with a constant $w$. This limit properly represents many relevant properties of the superconducting state. The functional integral over the size fluctuations of the scalar field can in this limit be approximated by the saddle point, and $H$ becomes, omitting irrelevant terms 


$$
H=\int d^{2} x\left[\frac{w^{2}}{2} \mathbf{p}_{\mathrm{s}}^{2}+\frac{1}{2}(\nabla \times \mathbf{A})^{2}\right]
$$

with $\mathbf{p}_{\mathrm{s}}$ the superfluid momentum

$$
\mathbf{p}_{\mathrm{s}}=\nabla \theta-2 e \mathbf{A}+\boldsymbol{\theta}^{\mathrm{P}}
$$

A so-called plastic field $\boldsymbol{\theta}^{\mathrm{P}}$ must be introduced for the following reason. The variable $\theta$ is a phase variable and is thus cyclic with periodicity $2 \pi$. A jump in $\theta$ by $2 \pi$ along a cut in the spatial plane indicates the presence of a point vortex. When this is the case the Hamiltonian requires a field $\boldsymbol{\theta}^{\mathrm{P}}$ to compensate for these jumps so that $H$ remains smooth. Such a construction with a compensating field is well known in the Villain formulation of lattice models, and it can also be given in the continuum [ 8 ]. The curl of $\boldsymbol{\theta}^{\mathrm{P}}$ gives the density of vortices consisting of $\delta$ functions at each vortex position

$$
\nabla \times \boldsymbol{\theta}^{\mathrm{P}}=2 \pi \sum_{\alpha} n_{\alpha} \delta\left(\mathbf{x}-\mathbf{x}_{\alpha}\right)
$$

where $n_{\alpha}$ is the winding number of a vortex, and $\mathbf{x}_{\alpha}$ its location. We will restrict ourselves to vortices with unit winding number, $n_{\alpha}= \pm 1$ for a vortex and antivortex, respectively. Since the theory is charged, vortices carry also a magnetic flux quantum $n_{\alpha} \pi / e$, and are more properly called magnetic vortices.

The solution of (4) is given by 8

$$
\theta_{i}^{\mathrm{P}}=-2 \pi \sum_{\alpha} n_{\alpha} \epsilon_{i j} \delta_{j}\left(\mathbf{x}, L_{\alpha}\right)
$$

where $\delta_{i}\left(\mathbf{x}, L_{\alpha}\right)$ is the $\delta$ function on the line $L_{\alpha}$ which starts at the position of the $\alpha$ th vortex $\mathbf{x}_{\alpha}$ and runs to spatial infinity along an arbitrary path:

$$
\delta_{i}\left(\mathbf{x}, L_{\alpha}\right)=\int_{\mathbf{x}_{\alpha}}^{\infty} d y_{i} \delta(\mathbf{x}-\mathbf{y})
$$

With the help of Stokes' theorem in two dimensions which can be expressed in differential form as $\nabla \cdot \boldsymbol{\delta}\left(\mathbf{x}, L_{\alpha}\right)=\delta\left(\mathbf{x}-\mathbf{x}_{\alpha}\right)$ it is easy to check that the explicit expression (5) for the

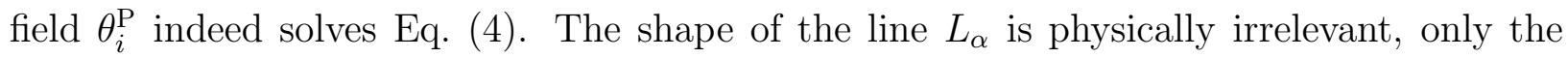


starting point which marks the position of the vortex matters. Leaving this point fixed the shape of $L_{\alpha}$ may be changed at will by performing the transformation

$$
\theta_{i}^{\mathrm{P}}(\mathbf{x}) \rightarrow \theta_{i}^{\mathrm{P}}(\mathbf{x})+\partial_{i} \delta(\mathbf{x}, S)
$$

where $\delta(\mathbf{x}, S)$ is the $\delta$ function on the surface $S$ swept out by $L_{\alpha}$ :

$$
\delta(\mathbf{x}, S):=\int_{S} d \sigma d \tau \epsilon_{i j} \partial_{\sigma} y_{i} \partial_{\tau} y_{j} \delta[\mathbf{x}-\mathbf{y}(\sigma, \tau)]
$$

with $\mathbf{y}(\sigma, \tau)$ being a parameterization of $S$. The superfluid momentum and thus the Hamiltonian is invariant under these so-called vortex gauge transformations [8].

For the time being vortices are ignored by setting the plastic field $\boldsymbol{\theta}^{\mathrm{P}}$ to zero. The partition function of the system is then given by

$$
Z=\int \mathcal{D} \theta \int \mathcal{D} \mathbf{A} \Xi(\mathbf{A}) \mathrm{e}^{-H}
$$

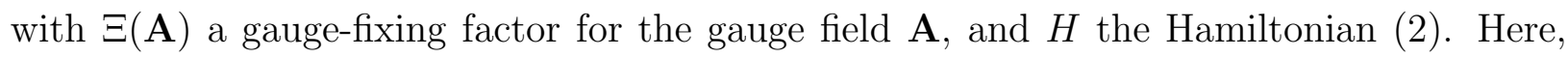
fields and coupling constants are rescaled so that no explicit temperature dependence appears in the Boltzmann factor. In (9) it is easy to integrate out the $\theta$ fluctuations. This yields for the partition function

$$
Z=\int \mathcal{D} \mathbf{A} \Xi(\mathbf{A}) \exp \left\{-\frac{1}{2} \int d^{2} x\left[(\nabla \times \mathbf{A})^{2}+m_{A}^{2} A_{i}\left(\delta_{i j}-\frac{\partial_{i} \partial_{j}}{\nabla^{2}}\right) A_{j}\right]\right\}
$$

where the last term with $m_{A}=2 e w$ is a gauge-invariant, albeit non-local mass term for the gauge field generated by the Higgs mechanism. A massless gauge field in $2 D$ represents no physical degrees of freedom. In Minkowski space-time, this is easily understood by recognizing that in $1+1$ dimensions there is no transverse direction. The system contains therefore only a single physical degree of freedom before the Higgs mechanism took place, namely $\theta$. This equals the number afterwards since a massive vector field represents only one independent degree of freedom in $2 D$. Note that the absence of genuine long-range order in $2 D$ is no obstacle to the Higgs mechanism. (The massless Schwinger model [12] provides the simplest soluble example for this.) 
We next introduce an auxiliary field $\mathrm{h}$ to linearize the first term in (10),

$$
\exp \left[-\frac{1}{2} \int d^{2} x(\nabla \times \mathbf{A})^{2}\right]=\int \mathcal{D} \mathrm{h} \exp \left[-\frac{1}{2} \int d^{2} x \mathrm{~h}^{2}+i \int d^{2} x \mathrm{~h} \cdot(\nabla \times \mathbf{A})\right]
$$

and integrate out the gauge-field fluctuations [after adding a gauge-fixing term $(1 / 2 \zeta)(\nabla$. A $)^{2}$. The result is a manifestly gauge-invariant expression for the partition function in terms of a massive scalar field $\mathrm{h}$, which represents the single degree of freedom contained in the theory:

$$
Z=\int \mathcal{D} \mathrm{h} \exp \left\{-\frac{1}{2} \int d^{2} x\left[\frac{1}{m_{A}^{2}}(\nabla \mathrm{h})^{2}+\mathrm{h}^{2}\right]\right\}
$$

To understand the physical significance of the $\mathrm{h}$ field appearing in this functional integral, we note that it follows from (11) that the auxiliary field h satisfies the equation

$$
\mathrm{h}=i \nabla \times \mathbf{A} .
$$

That is, the fluctuating field $\mathrm{h}$ coincides up to a factor $i$ with the local induction. Equation (12) shows that the magnetic field has a finite penetration depth $\lambda=1 / m_{A}$. In contrast to the Ginzburg-Landau description where the functional integral runs over the gauge potential, the integration variable in (12) is the physical field.

We now include vortex contributions. The mass $m_{A}$ provides the system with an infrared cutoff so that a single vortex in the charged theory has a finite energy, implying that there will always exist thermally activated vortices. This is different from the neutral model, describing a $2 D$ superfluid, where the absence of an infrared cutoff permits only tightly bound vortex-antivortex pairs to exist. We expect, accordingly, the superconducting phase to describe a plasma of vortices, each carrying one magnetic flux quantum $\pm \pi / e$. The partition function now reads

$$
Z=\sum_{n_{+}, n_{-}=0}^{\infty} \frac{z^{n_{+}+n_{-}}}{n_{+} ! n_{-} !} \int \prod_{\alpha} d^{2} x_{\alpha} \int \mathcal{D} \theta \int \mathcal{D} \mathbf{A} \exp \left\{-\frac{1}{2} \int d^{2} x\left[w^{2} \mathbf{p}_{\mathrm{s}}^{2}+(\nabla \times \mathbf{A})^{2}\right]\right\}
$$

where the superfluid momentum $\mathbf{p}_{\mathrm{s}}$ contains the vortex gauge field $\boldsymbol{\theta}^{\mathrm{P}}$, as in (3). In (14), $\int \prod_{\alpha} d^{2} x_{\alpha}$ denotes the integration over the positions of the vortices and the factor 
$1 / n_{+} !\left(1 / n_{-} !\right)$accounts for the fact that the (anti-)vortices are indistinguishable. The fugacity $z$ is the Boltzmann factor for an isolated vortex $z=\exp \left(-\epsilon_{\mathrm{c}}\right)$, with $\epsilon_{\mathrm{c}}$ being the vortex core energy.

The field $\boldsymbol{\theta}^{\mathrm{P}}$ can be shifted from the first to the second term in (14) by applying the transformation $\mathbf{A} \rightarrow \mathbf{A}-\boldsymbol{\theta}^{\mathrm{P}} /(2 e)$. This results in the shift

$$
\nabla \times \mathbf{A} \rightarrow \nabla \times \mathbf{A}-B^{\mathrm{P}}
$$

with the plastic field

$$
B^{\mathrm{P}}=-\frac{\pi}{e} \sum_{\alpha} n_{\alpha} \delta\left(\mathbf{x}-\mathbf{x}_{\alpha}\right)
$$

representing the magnetic flux density. Repeating the steps of the previous paragraph we now obtain instead of (12)

$$
Z=\sum_{n_{+}, n_{-}=0}^{\infty} \frac{z^{n_{+}+n_{-}}}{n_{+} ! n_{-} !} \int \prod_{\alpha} d \mathbf{x}_{\alpha} \int \mathcal{D} \mathrm{h} \exp \left\{-\frac{1}{2} \int d^{2} x\left[\frac{1}{m_{A}^{2}}(\nabla \mathrm{h})^{2}+\mathrm{h}^{2}\right]+i \int d^{2} x B^{\mathrm{P}} \mathrm{h}\right\}
$$

where $\mathrm{h}$ represents the physical local field

$$
\mathrm{h}=i\left(\nabla \times \mathbf{A}-B^{\mathrm{P}}\right)
$$

as will be clarified in a later section (Sec. V). The last term in (17) shows that the charge $g$ with which a magnetic vortex couples to the fluctuating $\mathrm{h}$ field is the product of an elementary flux quantum $\pi / e$ (which is contained in the definition of $B^{\mathrm{P}}$ ) and the inverse penetration depth $m_{A}$, i.e.,

$$
g=\frac{\pi}{e} m_{A}
$$

For small fugacities the summation indices $n_{+}$and $n_{-}$can be restricted to the values 0,1 and we arrive at the partition function of the massive sine-Gordon model [13]

$$
Z=\int \mathcal{D} \mathrm{h} \exp \left(-\int d^{2} x\left\{\frac{1}{2}\left[\frac{1}{m_{A}^{2}}(\nabla \mathrm{h})^{2}+\mathrm{h}^{2}\right]-2 N \cos \left(\frac{\pi}{e} \mathrm{~h}\right)\right\}\right) .
$$

This is the dual formulation of a $2 D$ superconductor. The vortices of unit winding number $n_{\alpha}= \pm 1$ turned the otherwise free theory (12) into an interacting one. 
The final form (20) demonstrates the basic concepts of a dual formulation: it is a formulation directly in terms of a (gauge-invariant) field representing the physical local induction. This is different from the Ginzburg-Landau description of a two-dimensional superconductor where the magnetic field is the curl of an unphysical gauge potential $\mathbf{A}$. The dual formulation also accounts for the topological excitations, viz., the magnetic vortices which in $2 D$ are point defects. They are coupled with a charge $g=(\pi / e) m_{A}$ to the magnetic field.

In three space dimensions these two basic ingredients will surface again. Since in $3 D$ the magnetic field has three components of which two are independent, the dual formulation will involve a massive vector field, rather than a simple massive scalar field $\mathrm{h}$ as in $2 D$. But what is more important, the point vortices of the two dimensional case become line defects in $3 D$. A grand canonical ensemble of closed loops requires, as will be demonstrated in the next section, a fluctuating field of its own to specify it. That is, accounting for the vortices does not simply add an interaction term to the theory without vortices, but adds a whole new theory. The coupling of this new theory — which turns out to be a complex $|\psi|^{4}$ theory-to the field representing the local field is again given by $(\pi / e) m_{A}$ as in two dimensions.

\section{FUNCTIONAL-INTEGRAL DESCRIPTION OF A LOOP GAS}

The subject of this section is the well-known fact that a loop gas can be described with the help of a functional integral involving a complex $|\psi|^{4}$ theory [8,14]. This will be used in a later section when formulating the dual theory of the Ginzburg-Landau theory in $3 D$. This theory features fluctuating closed magnetic vortices described by a disorder field theory. We will review here the derivation in the continuum, the discussion of the lattice derivation is relegated to the Appendix [8, 15].

Our point of departure is the correlation function $G(\mathbf{x})$ of a free complex field theory defined by the Hamiltonian

$$
H=\int d^{3} x\left(|\nabla \psi|^{2}+m^{2}|\psi|^{2}\right)
$$

Explicitly, 


$$
G(\mathbf{x})=\int \frac{d^{3} k}{(2 \pi)^{3}} \frac{\mathrm{e}^{i \mathbf{k} \cdot \mathbf{x}}}{\mathbf{k}^{2}+m^{2}}
$$

This is written in the Schwinger proper-time representation as an integral over the proper time $s$

$$
\begin{aligned}
G(\mathbf{x}) & =\int_{0}^{\infty} d s \mathrm{e}^{-s m^{2}} \int \frac{d^{3} k}{(2 \pi)^{3}} \mathrm{e}^{i \mathbf{k} \cdot \mathbf{x}} \mathrm{e}^{-s \mathbf{k}^{2}} \\
& =\int_{0}^{\infty} d s \mathrm{e}^{-s m^{2}}\left(\frac{1}{4 \pi s}\right)^{3 / 2} \mathrm{e}^{-\frac{1}{4} \mathbf{x}^{2} / s},
\end{aligned}
$$

where we used the identity

$$
\frac{1}{a}=\int_{0}^{\infty} d s \mathrm{e}^{-s a}
$$

According to Feynman's formulation of quantum mechanics [11 the right-hand side of (23) can be represented as a sum over all real-space paths of a particle with mass $\frac{1}{2}$ running from 0 at imaginary time 0 to $\mathbf{x}$ at time $s$ :

$$
\left(\frac{1}{4 \pi s}\right)^{3 / 2} \mathrm{e}^{-\frac{1}{4} \mathbf{x}^{2} / s}=\int_{\mathbf{x}(0)=0}^{\mathbf{x}(s)=\mathbf{x}} \mathcal{D} \mathbf{x}\left(s^{\prime}\right) \exp \left[-\frac{1}{4} \int_{0}^{s} d s^{\prime} \dot{\mathbf{x}}^{2}\left(s^{\prime}\right)\right],
$$

involving the free "Lagrangian" $L=\dot{\mathbf{x}}^{2} / 4$. The extra Boltzmann factor $\exp \left(-s m^{2}\right)$ in (23) suppresses large proper-time values exponentially, and the integral $\int_{0}^{\infty} d s$ shows that all positive values of $s$ are allowed and accounted for.

From integrating the identity (24) with respect to $a$ it follows that to within an additive constant

$$
\ln (a)=-\int_{0}^{\infty} \frac{d s}{s} \mathrm{e}^{-s a}
$$

Employing this we can use the previous result to write

$$
\begin{aligned}
\operatorname{Tr} \ln \left(-\nabla^{2}+m^{2}\right) & =-\int_{0}^{\infty} \frac{d s}{s} \mathrm{e}^{-s m^{2}} \int \frac{d^{3} k}{(2 \pi)^{3}} \mathrm{e}^{-s \mathbf{k}^{2}} \\
& =-\int_{0}^{\infty} \frac{d s}{s} \mathrm{e}^{-s m^{2}} \oint \mathcal{D} \mathbf{x}\left(s^{\prime}\right) \exp \left[-\frac{1}{4} \int_{0}^{s} d s^{\prime} \dot{\mathbf{x}}^{2}\left(s^{\prime}\right)\right],
\end{aligned}
$$

where $\operatorname{Tr}$ denotes the integral over the momentum variables 


$$
\operatorname{Tr} \ln \left(-\nabla^{2}+m^{2}\right)=\int \frac{d^{3} k}{(2 \pi)^{3}} \ln \left(\mathbf{k}^{2}+m^{2}\right)
$$

The path integral $\oint \mathcal{D} \mathbf{x}\left(s^{\prime}\right)$ in (27) runs over closed loops starting and ending in 0 . Using the identity

$$
\operatorname{Tr} \ln \left(-\nabla^{2}+m^{2}\right)=\ln \operatorname{Det}\left(-\nabla^{2}+m^{2}\right),
$$

we can write the inverse determinant as

$$
\operatorname{Det}^{-1}\left(-\nabla^{2}+m^{2}\right)=\exp \left(-W_{0}\right)
$$

with $W_{0}$ denoting the right-hand side of (27). The exponential $\exp \left(-W_{0}\right)$ has the expansion

$$
\mathrm{e}^{-W_{0}}=\sum_{N=0}^{\infty} \frac{1}{N !} \prod_{l=1}^{N}\left[\int_{0}^{\infty} \frac{d s_{l}}{s_{l}} \mathrm{e}^{-m^{2} s_{l}} \oint \mathcal{D} \mathbf{x}\left(s_{l}^{\prime}\right)\right] \exp \left[-\frac{1}{4} \sum_{l=1}^{N} \int_{0}^{s_{l}} d s_{l}^{\prime} \dot{\mathbf{x}}^{2}\left(s_{l}^{\prime}\right)\right],
$$

which we recognize as the partition function $Z$ of a grand canonical ensemble of fluctuating closed oriented random loops, of arbitrary length and shape. We will refer to such an ensemble as loop gas. On account of (30), the exponential $\exp \left(-W_{0}\right)$ may alternatively be viewed as an inverse functional determinant. As such it is easily recognized as the partition function of the free complex field theory,

$$
Z=\int \mathcal{D} \psi^{*} \mathcal{D} \psi \mathrm{e}^{-H}
$$

with $H$ the Hamiltonian (21). Thus we have proved the equivalence of a free complex field theory and a free loop gas.

In a superconductor, the vortices have short-range interactions. To describe these we start again from the field theoretic side. We shall prove that the Hamiltonian

$$
H=\int d^{3} x\left[|\nabla \psi|^{2}+m^{2}|\psi|^{2}+\lambda\left(|\psi|^{2}\right)^{2}\right]
$$

with the additional interaction term, accounts for the steric repulsion in the loop gas. For this we write the complex field $\psi$ as $\psi=\left(\psi_{1}+i \psi_{2}\right) / \sqrt{2}$ and express the interaction term as a functional integral over an auxiliary field $\sigma$ 


$$
\exp \left[-\frac{\lambda}{4} \int d^{3} x\left(\psi_{a}^{2}\right)^{2}\right]=\int \mathcal{D} \sigma \exp \left[-\int d^{3} x\left(\frac{1}{\lambda} \sigma^{2}-i \psi_{a}^{2} \sigma\right)\right]
$$

where the index $a$ runs over 1,2 . The partition function becomes now

$$
\begin{aligned}
Z= & \int \mathcal{D} \psi_{1} \mathcal{D} \psi_{2} \mathcal{D} \sigma \exp \left\{-\int d^{3} x\left[\frac{1}{2}\left(\nabla \psi_{a}\right)^{2}+\frac{m^{2}}{2} \psi_{a}^{2}-i \sigma \psi_{a}^{2}+\frac{1}{\lambda} \sigma^{2}\right]\right\} \\
= & \int \mathcal{D} \sigma \operatorname{Det}^{-1}\left(-\nabla^{2}+m^{2}-2 i \sigma\right) \exp \left(-\frac{1}{\lambda} \int d^{3} x \sigma^{2}\right) \\
= & \int \mathcal{D} \sigma \exp \left(-\frac{1}{\lambda} \int d^{3} x \sigma^{2}\right) \sum_{N=0}^{\infty} \frac{1}{N !} \prod_{l=1}^{N}\left[\int_{0}^{\infty} \frac{d s_{l}}{s_{l}} \mathrm{e}^{-m^{2} s_{l}} \oint \mathcal{D} \mathbf{x}\left(s_{l}^{\prime}\right)\right] \\
& \times \exp \left(-\sum_{l=1}^{N} \int_{0}^{s_{l}} d s_{l}^{\prime}\left\{\frac{1}{4} \dot{\mathbf{x}}^{2}\left(s_{l}^{\prime}\right)-2 i \sigma\left[\mathbf{x}\left(s_{l}^{\prime}\right)\right]\right\}\right),
\end{aligned}
$$

where the last equality follows from the previous result (31). A simple Gaussian integration yields

$$
\int \mathcal{D} \sigma \exp \left\{-\frac{1}{\lambda} \int d^{3} x \sigma^{2}+2 i \int_{0}^{s} d s^{\prime} \sigma\left[\mathbf{x}\left(s^{\prime}\right)\right]\right\}=\exp \left\{-\lambda \int_{0}^{s} d s^{\prime} d s^{\prime \prime} \delta\left[\mathbf{x}\left(s^{\prime}\right)-\mathbf{x}\left(s^{\prime \prime}\right)\right]\right\}
$$

Using this in the last expression for $Z$, we obtain the real-space representation for the partition function of a complex $|\psi|^{4}$ theory

$$
\begin{aligned}
& Z=\sum_{N=0}^{\infty} \frac{1}{N !} \prod_{l=1}^{N}\left[\int_{0}^{\infty} \frac{d s_{l}}{s_{l}} \mathrm{e}^{-m^{2} s_{l}} \oint \mathcal{D} \mathbf{x}\left(s_{l}^{\prime}\right)\right] \exp \left\{-\frac{1}{4} \sum_{l=1}^{N} \int_{0}^{s_{l}} d s_{l}^{\prime} \dot{\mathbf{x}}^{2}\left(s_{l}^{\prime}\right)\right. \\
&\left.-\lambda \sum_{l, k=1}^{N} \int_{0}^{s_{l}} d s_{l}^{\prime} \int_{0}^{s_{k}} d s_{k}^{\prime \prime} \delta\left[\mathbf{x}\left(s_{l}^{\prime}\right)-\mathbf{x}\left(s_{k}^{\prime \prime}\right)\right]\right\} .
\end{aligned}
$$

This is recognized as the partition function of a loop gas with short-range repulsion. The field theoretic representation of an interacting loop gas by a $|\psi|^{4}$ field theory will be employed in Sec. $\mathrm{Z}$ to formulate the dual theory of a $3 D$ superconductor.

\section{DUAL TRANSFORMATION OF A $3 D$ LATTICE SUPERCONDUCTOR}

In this section we set up a dual formulation of a $3 D$ superconductor on a lattice. In contrast to the continuum case to be discussed in the next section, the lattice model can be transformed exactly to its dual version. We take as starting point the partition function [3] 


$$
Z=\prod_{\mathbf{x}}\left[\int d \mathbf{A}(\mathbf{x}) \delta(\bar{\nabla} \cdot \mathbf{A})\right] \mathrm{e}^{-\frac{1}{2} \sum_{\mathbf{x}}(\bar{\nabla} \times \mathbf{A})^{2}} \prod_{\mathbf{x}}\left[\int_{\pi / a}^{\pi / a} \frac{d \theta(\mathbf{x})}{2 \pi}\right] \exp \left\{\beta \sum_{i, \mathbf{x}} \cos \left[\partial_{i} \theta(\mathbf{x})-q A_{i}(\mathbf{x})\right]\right\}
$$

where $\theta(\mathbf{x})$ is a phase variable at site $\mathbf{x}$ of a $3 D$ cubic lattice, $\mathbf{A}(\mathbf{x})$ is the electromagnetic gauge potential which is a real non-compact variable defined on the directed links between adjacent sites, $\beta$ is the inverse temperature, and $q$ is the electric charge. Moreover, $\mathbf{x}=a x_{i} \mathbf{i}$, with $a$ the lattice spacing, $x_{i}$ integers labeling the sites, and $\mathbf{i}$ three orthogonal vectors spanning the lattice. The sum $\sum_{\mathbf{x}}$, which includes a factor $a^{3}$, runs over all lattice sites, while the sum $\sum_{i}$ runs over all directions; $\nabla$ is a lattice derivative with components

$$
\partial_{i} f(\mathbf{x})=\frac{1}{a}[f(\mathbf{x}+a \mathbf{i})-f(\mathbf{x})] ; \quad \bar{\partial}_{i} f(\mathbf{x})=\frac{1}{a}[f(\mathbf{x})-f(\mathbf{x}-a \mathbf{i})]
$$

and the delta function $\delta(\bar{\nabla} \cdot \mathbf{A})$ in (38) fixes the gauge. The lattice model (38) is appropriate for a non-compact gauge group. We shall be working in the Villain approximation [17] of the model, which is obtained by the following replacement

$$
\begin{aligned}
Z_{x y} & =\prod_{\mathbf{x}}\left[\int_{\pi / a}^{\pi / a} \frac{d \theta(\mathbf{x})}{2 \pi}\right] \exp \left\{\beta \sum_{i, \mathbf{x}} \cos \left[\partial_{i} \theta(\mathbf{x})-q A_{i}(\mathbf{x})\right]\right\} \\
& \rightarrow \prod_{\mathbf{x}}\left[\int_{\pi / a}^{\pi / a} \frac{d \theta(\mathbf{x})}{2 \pi}\right] \sum_{\{\mathbf{n}(\mathbf{x})\}} \exp \left\{-\frac{\beta}{2} \sum_{\mathbf{x}}[\nabla \theta(\mathbf{x})-q \mathbf{A}(\mathbf{x})-2 \pi \mathbf{n}(\mathbf{x})]^{2}\right\},
\end{aligned}
$$

where $\mathbf{n}(\mathbf{x})$ are integers which are - like the gauge potential $\mathbf{A}(\mathbf{x})$-defined on the directed links between adjacent sites. The partition function in (40) is given the index $x y$ to indicate that this part of the theory is simply the $x y$ model when the electric charge $q$ is set to zero. We proceed by introducing an auxiliary field $\mathbf{v}(\mathbf{x})$ via a quadratic completion

$$
\begin{aligned}
Z_{x y}=\prod_{\mathbf{x}}[ & \left.\int_{\pi / a}^{\pi / a} \frac{d \theta(\mathbf{x})}{2 \pi} \int d \mathbf{v}(\mathbf{x})\right] \\
& \times \sum_{\{\mathbf{n}(\mathbf{x})\}} \exp \left\{-\frac{1}{2 \beta} \sum_{\mathbf{x}} \mathbf{v}^{2}(\mathbf{x})+i \sum_{\mathbf{x}} \mathbf{v}(\mathbf{x}) \cdot[\nabla \theta(\mathbf{x})-q \mathbf{A}(\mathbf{x})-2 \pi \mathbf{n}(\mathbf{x})]\right\} .
\end{aligned}
$$

The integration over $\theta(\mathbf{x})$ can then be carried out to yield the constraint $\bar{\nabla} \cdot \mathbf{v}(\mathbf{x})=0$, while the sum $\sum_{\{\mathbf{n}(\mathbf{x})\}} \exp [-2 \pi i \mathbf{v}(\mathbf{x}) \cdot \mathbf{n}(\mathbf{x})]$ forces the integral over the real variable $\mathbf{v}$ to take on only integer values $\mathbf{l}$, say. This last observation follows from the Poisson summation formula 


$$
\sum_{n} \mathrm{e}^{-2 \pi i v n}=\sum_{l} \delta(v-l)
$$

In this way the partition function $Z_{x y}$ becomes:

$$
Z_{x y}=\sum_{\{\mathbf{l}(\mathbf{x})\}} \delta_{\bar{\nabla} \cdot \mathbf{l}, 0} \exp \left[-\frac{1}{2 \beta} \sum_{\mathbf{x}} \mathbf{l}^{2}(\mathbf{x})-i q \sum_{\mathbf{x}} \mathbf{l}(\mathbf{x}) \cdot \mathbf{A}(\mathbf{x})\right] .
$$

Whereas representation (40) of $Z_{x y}$ is a field theoretic description of the lattice model, Eq. (43) describes it in the real-space language of (charged) closed loops. This is analogous to the twofold description of a loop gas in the continuum which we discussed in the preceding section.

The constraint $\bar{\nabla} \cdot \mathbf{l}=0$ in (43) can be explicitly solved by introducing an auxiliary integer-valued potential $\mathbf{i}(\mathbf{x})$, via $\mathbf{l}=\bar{\nabla} \times \mathbf{i}$, so that

$$
Z_{x y}=\prod_{\mathbf{x}}\left[\int d \mathbf{h}(\mathbf{x}) \delta(\bar{\nabla} \cdot \mathbf{h})\right] \sum_{\{\mathbf{m}(\mathbf{x})\}} \delta_{\bar{\nabla} \cdot \mathbf{m}, 0} \mathrm{e}^{-2 \pi i \sum_{\mathbf{x}} \mathbf{m} \cdot \mathbf{h}} \exp \left[-\frac{1}{2 \beta} \sum_{\mathbf{x}}(\bar{\nabla} \times \mathbf{h})^{2}-i q \sum_{\mathbf{x}}(\bar{\nabla} \times \mathbf{h}) \cdot \mathbf{A}\right] .
$$

Here, because of the presence of the factor $\exp \left(-2 \pi i \sum_{\mathbf{x}} \mathbf{m} \cdot \mathrm{h}\right)$, the sum over the integervalued fields $\mathbf{m}(\mathbf{x})$ ensures, on account of the Poisson formula (42), that only integer-valued fields $\mathrm{h}(\mathbf{x})=\mathbf{i}(\mathbf{x})$ contribute to the integral $\int d \mathbf{h}(\mathbf{x})$, as required. For $q=0$, corresponding to the uncharged $x y$ model, Eq. (44) gives a dual representation in terms of vortices $\mathbf{m}$ coupled to a massless vector field $\mathrm{h}$. The condition $\bar{\nabla} \cdot \mathbf{m}=0$ in (44) is to assure that the vortices form closed loops, which is physically the case. When the integration over the field $\mathrm{h}$ is carried out, so that one is only left with vortices, one finds them interacting via long-range forces of the Biot-Savart type.

For the lattice superconductor we have to add the electromagnetic part to $Z_{x y}$ [4]:

$$
\begin{aligned}
Z= & \prod_{\mathbf{x}}\left[\int d \mathbf{A}(\mathbf{x}) \delta(\bar{\nabla} \cdot \mathbf{A}) \int d \mathbf{h}(\mathbf{x}) \delta(\bar{\nabla} \cdot \mathbf{h})\right] \sum_{\{\mathbf{m}(\mathbf{x})\}} \delta_{\bar{\nabla} \cdot \mathbf{m}, 0} \\
& \times \exp \left[-\frac{1}{2} \sum_{\mathbf{x}}(\bar{\nabla} \times \mathbf{A})^{2}-2 \pi i \sum_{\mathbf{x}} \mathbf{m} \cdot \mathbf{h}-\frac{1}{2 \beta} \sum_{\mathbf{x}}(\bar{\nabla} \times \mathbf{h})^{2}-i q \sum_{\mathbf{x}}(\bar{\nabla} \times \mathbf{h}) \cdot \mathbf{A}\right] \\
= & \prod_{\mathbf{x}}\left[\int d \mathbf{h}(\mathbf{x}) \delta(\bar{\nabla} \cdot \mathbf{h})\right] \sum_{\{\mathbf{m}(\mathbf{x})\}} \delta_{\bar{\nabla} \cdot \mathbf{m}, 0} \mathrm{e}^{-2 \pi i \sum_{\mathbf{x}} \mathbf{m} \cdot \mathbf{h}} \exp \left\{-\frac{1}{2 \beta} \sum_{\mathbf{x}}\left[(\bar{\nabla} \times \mathbf{h})^{2}+\beta q^{2} \mathbf{h}^{2}\right]\right\},
\end{aligned}
$$


where in the last step we carried out the integral over the electromagnetic gauge potential which resulted in a mass term for the vector field $\mathrm{h}$. The constraint $\nabla \mathrm{h}=0$ is an intrinsic part of the description of a fluctuating massive vector field. For a non-fluctuating field it follows automatically from the field equation. Physically, (45) represents a loop gas of vortices coupled to a massive vector field. The massiveness of $\mathrm{h}$ is in fact the only difference with the dual description of the $x y$ model, Eq. (44) with $q=0$. As a result, when integrating out this vector field, one obtains again a Biot-Savart type of force between the vortices, but now of finite range.

In the next section, an equivalent dual map will be carried out in the continuum, yielding a continuum version of the lattice model (45). The fluctuating vector field $\mathrm{h}$ will be related to the physical local induction. With this identification in mind the constraint $\bar{\nabla} \cdot \mathrm{h}=0$ in (44) can be understood as representing the fact that the magnetic induction is divergence-free.

\section{DUAL MAP OF A $3 D$ SUPERCONDUCTOR}

This section is the central part of the paper, in which we derive the dual formulation of the Ginzburg-Landau model. We shall argue that this description is one in terms of a genuine order parameter which involves a global rather than a local symmetry as is the case in the Ginzburg-Landau formulation [9]. For this reason the dual theory can be employed to arrive at a conventional Landau description of the superconducting phase transition [8,18,19], which will be the subject of Sec. [X].

The fundamental object of the dual description is the magnetic vortex, or Abrikosov flux tube. Such a topological defect is either closed, infinitely long, or - in the case of a finite system - starts and ends at the boundary of the specimen. A magnetic vortex can never terminate inside a superconductor. However, for reasons that shall become clear when we proceed, we will employ a construct that allows us to describe, at least theoretically, magnetic vortices that do terminate inside the superconductor. To this end we allow the system to contain a Dirac monopole [20] as a test particle. Recall that due to the Meissner 
effect, a magnetic field can penetrate a superconductor only by forming quantized flux tubes. Since also the flux lines emanating from the monopole are squeezed into a tube, a monopole produces precisely such a vortex.

Section 1 revealed that a magnetic vortex is described by a plastic field $\mathbf{B}^{\mathrm{P}}$ which appears in the theory in the combination $\nabla \times \mathbf{A}-\mathbf{B}^{\mathrm{P}}$ with the gauge field [20]. In other words, in the presence of a test tube, the Hamiltonian becomes

$$
H^{\mathrm{P}}=\int d^{3} x\left[\frac{1}{2}\left(\nabla \times \mathbf{A}-\mathbf{B}^{\mathrm{P}}\right)^{2}+\frac{1}{2} m_{A}^{2} A_{i}\left(\delta_{i j}-\frac{\partial_{i} \partial_{j}}{\nabla^{2}}\right) A_{j}+\frac{1}{2 \zeta}(\nabla \cdot \mathbf{A})^{2}\right],
$$

where we added a gauge-fixing term $(1 / 2 \zeta)(\nabla \cdot \mathbf{A})^{2}$, and given $H$ the superscript $\mathrm{P}$ to indicate the presence of the test particle with its emanating tube. We recall that we consider the Ginzburg-Landau model (11) in the London limit, where the superconducting order field is written as $\phi(x)=\exp [i \theta(x)] w / \sqrt{2}$, with $w$ a constant. The mass term, with $m_{A}=2 e w$, is a result of integrating out the phase field $\theta$. In three dimensions, the plastic field $\mathbf{B}^{\mathrm{P}}$ has the form [20,21]

$$
B_{i}^{\mathrm{P}}(\mathbf{x})=\frac{\pi}{e} \int_{L_{\mathbf{z}}} d s \frac{d y_{i}}{d s} \delta[\mathbf{x}-\mathbf{y}(s)]=\frac{\pi}{e} \int_{L_{\mathbf{z}}} d y_{i} \delta(\mathbf{x}-\mathbf{y}),
$$

which is the proper three-dimensional generalization of the $2 D$ result (116). The field satisfies the equation $\nabla \cdot \mathbf{B}^{\mathrm{P}}(\mathbf{x})=(\pi / e) \delta(\mathbf{x}-\mathbf{z})$ on account of Stokes' theorem, implying that it indeed describes a monopole located at $\mathbf{z}$. The line $L_{\mathbf{z}}$ starting at the point $\mathbf{z}$ and running to infinity is the Dirac string accompanying the monopole. As will be clarified below, the

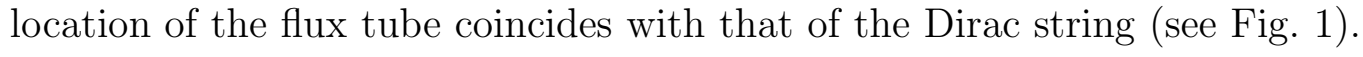

From (46) we infer that the operator $V\left(L_{\mathbf{z}}\right)$ describing the test tube is given by

$$
V\left(L_{\mathbf{z}}\right)=\mathrm{e}^{-\frac{1}{2} \int d^{3} x\left(\mathbf{B}^{\mathrm{P}}\right)^{2}} \exp \left[\int d^{3} x(\nabla \times \mathbf{A}) \cdot \mathbf{B}^{\mathrm{P}}\right] .
$$

We are interested in the expectation value of this operator:

$$
\left\langle V\left(L_{\mathbf{z}}\right)\right\rangle=\int \mathcal{D} \mathbf{A} \mathrm{e}^{-H^{\mathrm{P}}}
$$

Since the integral over $\mathbf{A}$ is Gaussian, it can be evaluated by substituting the field equation, 


$$
A_{i}(\mathbf{x})=\int d^{3} y \Delta_{i j}(\mathbf{x}-\mathbf{y})\left[\nabla \times \mathbf{B}^{\mathrm{P}}(\mathbf{y})\right]_{j}
$$

back into the exponent. The gauge-field correlation function $\Delta_{i j}$ appearing here is

$$
\Delta_{i j}(\mathbf{x})=\int \frac{d^{3} k}{(2 \pi)^{3}}\left(\frac{\delta_{i j}-\left(k_{i} k_{j}\right) / \mathbf{k}^{2}}{\mathbf{k}^{2}+m_{A}^{2}}+\zeta \frac{k_{i} k_{j}}{\mathbf{k}^{4}}\right) \mathrm{e}^{i \mathbf{k} \cdot \mathbf{x}}
$$

The gauge-dependent longitudinal part of the correlation function does not contribute to (49) since $\nabla \cdot\left(\nabla \times \mathbf{B}^{\mathrm{P}}\right)=0$. The expectation value is therefore independent of gauge choice.

The local induction corresponding to the classical solution (50) in the presence of the test tube is

$$
\nabla \times \mathbf{A}(\mathbf{x})-\mathbf{B}^{\mathrm{P}}(\mathbf{x})=\frac{\pi}{e} \nabla \Delta(\mathbf{x}-\mathbf{z})-m_{A}^{2} \int d^{3} y \Delta(\mathbf{x}-\mathbf{y}) \mathbf{B}^{\mathrm{P}}(\mathbf{y}),
$$

where

$$
\Delta(\mathbf{x})=\int \frac{d^{3} k}{(2 \pi)^{3}} \frac{\mathrm{e}^{i \mathbf{k} \cdot \mathbf{x}}}{\mathbf{k}^{2}+m_{A}^{2}}=\frac{\mathrm{e}^{-m_{A}|\mathbf{x}|}}{4 \pi|\mathbf{x}|}
$$

is the Yukawa potential. The term $\mathbf{B}^{\mathrm{P}}$ in (52) describes the Dirac string $L_{\mathbf{z}}$, the first term on the right-hand side corresponds to the screened Coulomb force generated by the monopole. The last term, which is only present when $m_{A} \neq 0$-i.e., when there is a Meissner effectdescribes the magnetic flux tube. A closer inspection of (52) reveals that the subtraction of the Dirac string $\mathbf{B}^{\mathrm{P}}$ from the field $\nabla \times \mathbf{A}$ is necessary in order to obtain the physical local induction $\mathbf{h}$ 20,21. Indeed, if we calculate from the right-hand side of (52) the magnetic flux through a plane perpendicular to the Dirac string, we find

$$
\int d^{2} x_{i}\left[\frac{\pi}{e} \partial_{i} \Delta(\mathbf{x}-\mathbf{z})-m_{A}^{2} \int d^{3} y \Delta(\mathbf{x}-\mathbf{y}) B_{i}^{\mathrm{P}}(\mathbf{y})\right]=-\frac{\pi}{e}
$$

that precisely one flux quantum pierces the surface in the negative direction (see Fig. 1). Here, $d^{2} x_{i}$ is an element of the surface orthogonal to the Dirac string, and we used Gauss' law to rewrite the first term on the left-hand side as

$$
\frac{\pi}{e} \int d^{2} x_{i} \partial_{i} \Delta(\mathbf{x}-\mathbf{z})=\frac{\pi}{e} \int d^{3} x \nabla^{2} \Delta(\mathbf{x}-\mathbf{z})
$$


Equation (54) confirms Dirac's statement that the magnetic flux emanating from a monopole must be supplied by an infinitesimally thin string of magnetic dipoles and that in order to obtain the true local field of a genuine point monopole, this string has to be subtracted. While this string is indeed completely unphysical in the normal phase, it acquires a physical relevance in the superconducting phase [22] where it serves as the core of the Abrikosov flux tube.

Substituting the field equation (50) back into the theory, we obtain for the vacuum expectation value $\left\langle V\left(L_{\mathbf{z}}\right)\right\rangle$ in the London limit

$$
\left\langle V\left(L_{\mathbf{z}}\right)\right\rangle=\mathrm{e}^{\frac{1}{2} \int d^{3} x\left(\mathbf{B}^{\mathrm{P}}\right)^{2}} \exp \left\{\frac{1}{2} \int d^{3} x d^{3} y\left[\nabla \times \mathbf{B}^{\mathrm{P}}(\mathbf{x})\right]_{i} \Delta_{i j}(\mathbf{x}-\mathbf{y})\left[\nabla \times \mathbf{B}^{\mathrm{P}}(\mathbf{y})\right]_{j}\right\},
$$

or

$$
\left\langle V\left(L_{\mathbf{z}}\right)\right\rangle=\exp \left\{-\frac{1}{2} \int d^{3} x d^{3} y\left[\rho(\mathbf{x}) \Delta(\mathbf{x}-\mathbf{y}) \rho(\mathbf{y})+m_{A}^{2} B_{i}^{\mathrm{P}}(\mathbf{x}) \Delta(\mathbf{x}-\mathbf{y}) B_{i}^{\mathrm{P}}(\mathbf{y})\right]\right\}
$$

where $\rho(\mathbf{x})=(\pi / e) \delta(\mathbf{x}-\mathbf{z})$ is the monopole density. In deriving this we have omitted terms depending only on $w$.

It should be noted that the first factor in (56) diverges

$$
\frac{1}{2} \int d^{3} x\left(\mathbf{B}^{\mathrm{P}}\right)^{2}=\frac{1}{2}\left(\frac{\pi}{e}\right)^{2} \int_{L_{\mathbf{z}}} d x_{i} \int_{L_{\mathbf{z}}} d y_{i} \delta(\mathbf{x}-\mathbf{y}),
$$

representing the self-interaction of the Dirac string. This term canceled in (57). The first term in (57) contains for $\mathbf{x}=\mathbf{y}$ a diverging monopole self-interaction. This divergence is irrelevant and can be eliminated by defining a renormalized operator

$$
V_{\mathrm{r}}\left(L_{\mathbf{z}}\right)=V\left(L_{\mathbf{z}}\right) \exp \left[\frac{\pi^{2}}{2 e^{2}} \Delta(0)\right] \text {. }
$$

The second term in (57) is the most important one for our purposes. It represents a BiotSavart interaction between two line elements $d x_{i}$ and $d y_{i}$ of the magnetic vortex (see Fig. 2). It contains an ultraviolet singularity due to fact that in the London limit, where the mass $\left|m_{\phi}\right|$ of the superconducting order field is taken to be infinite, the vortices are considered to be ideal lines. For a finite mass the magnetic vortices have a typical width of the order of 
the coherence length $\xi=1 /\left|m_{\phi}\right|$. This mass therefore provides a natural ultraviolet cutoff to the theory. The last term in (57) then takes the form 22

$$
-\frac{m_{A}^{2}}{2}\left(\frac{\pi}{e}\right)^{2} \int_{L_{\mathbf{z}}} d x_{i} \int_{L_{\mathbf{z}}} d y_{i} \Delta(\mathbf{x}-\mathbf{y})=-M_{V}\left|L_{\mathbf{z}}\right|
$$

with $\left|L_{\mathbf{z}}\right|$ the (infinite) length of the flux tube, and [23]

$$
M_{V}=\left(\frac{\pi}{e}\right)^{2} \frac{m_{A}^{2}}{8 \pi} \ln \left(\frac{\left|m_{\phi}^{2}\right|}{m_{A}^{2}}\right)=\frac{\pi w^{2}}{2} \ln \left(\frac{\lambda^{2}}{\xi^{2}}\right)
$$

being the free energy per unit length. The combination $\lambda / \xi$ defines the dimensionless Ginzburg-Landau parameter $\kappa$, which in the London limit is much larger than 1.

For a monopole-antimonopole pair, (60) amounts to a confining linear potential between the monopole and antimonopole in the superconducting phase. Let $V^{*}\left(L_{\overline{\mathbf{z}}}\right)$ describe an antimonopole located at $\overline{\mathbf{z}}$, with $L_{\overline{\mathbf{z}}}$ being a line running from infinity to $\overline{\mathbf{z}}$. Collecting all terms, we find for such a pair

$$
\left\langle V_{\mathrm{r}}\left(L_{\mathbf{z}}\right) V_{\mathrm{r}}^{*}\left(L_{\overline{\mathbf{z}}}\right)\right\rangle=\exp \left(-M_{V}\left|L_{\mathbf{z} \overline{\mathbf{z}}}\right|\right) \exp \left(\frac{\pi}{4 e^{2}} \frac{\mathrm{e}^{-m_{A}\left|L_{\mathbf{z} \overline{\mathbf{z}}}\right|}}{\left|L_{\mathbf{z} \overline{\mathbf{z}}}\right|}\right),
$$

where $L_{\mathbf{z} \overline{\mathbf{z}}}$ is the flux tube connecting the monopole at $\mathbf{z}$ with the antimonopole at $\overline{\mathbf{z}}$, and $\left|L_{\mathbf{z} \overline{\mathbf{z}}}\right|$ is its length.

We remark that the two Dirac strings may initially run to any point at infinity. Due to the string tension, they join on the shortest path $L_{\mathbf{z} \overline{\mathbf{z}}}$ between the monopoles. The result (62) is central to our line of arguments. It shows that the correlation function $\left\langle V_{\mathrm{r}}\left(L_{\mathbf{z}}\right) V_{\mathrm{r}}^{*}\left(L_{\overline{\mathbf{z}}}\right)\right\rangle$ behaves differently in the two phases [9,24]. In the superconducting phase, where the gauge field is massive, the "confinement" factor dominates and the correlation function decays exponentially for distances larger than $1 / M_{V}$ :

$$
\left\langle V_{\mathrm{r}}\left(L_{\mathbf{z}}\right) V_{\mathrm{r}}^{*}\left(L_{\overline{\mathbf{z}}}\right)\right\rangle \stackrel{\left|L_{\mathbf{z} \overline{\mathbf{z}}}\right| \rightarrow \infty}{\longrightarrow} 0
$$

This behavior is typical for an operator in a phase without massless excitations. On the other hand, in the high-temperature phase, where the gauge field is massless, the confinement factor in the correlation function (62) disappears, while the argument of the second exponential 
turns into a pure Coulomb potential. The correlation function remains, consequently, finite for large distances:

$$
\left\langle V_{\mathrm{r}}\left(L_{\mathbf{z}}\right) V_{\mathrm{r}}^{*}\left(L_{\overline{\mathbf{z}}}\right)\right\rangle \stackrel{\left|L_{\mathbf{z}}\right| \rightarrow \infty}{\longrightarrow} 1
$$

By the cluster property of correlation functions this implies that the operator describing the test tube develops a vacuum expectation value. This signals a proliferation of magnetic vortices. Indeed, according to (61) the free energy $M_{V}$ per unit length of a vortex vanishes at the transition point, where $w \rightarrow 0$. It should be noted that it is the high-temperature phase and not the superconducting phase where $V_{\mathrm{r}}\left(L_{\mathbf{z}}\right)$ develops an expectation value.

Before deriving the full-fledged dual theory let us rederive the correlation function (62) in a way that reveals some aspects of the nature of the dual theory. To this end we linearize the functional integral over the gauge field by introducing an auxiliary field $h$. In the gauge $\nabla \cdot \mathbf{A}=0$, which corresponds to setting $\zeta=0$, we find

$$
\left\langle V_{\mathrm{r}}\left(L_{\mathbf{z}}\right) V_{\mathrm{r}}^{*}\left(L_{\overline{\mathbf{z}}}\right)\right\rangle=\int \mathcal{D} \mathbf{A} \mathcal{D} \mathrm{h} \exp \left[-\frac{1}{2} \int d^{3} x \mathrm{~h}^{2}+i \int d^{3} x \mathrm{~h} \cdot\left(\nabla \times \mathbf{A}-\mathbf{B}^{\mathrm{P}}\right)-\frac{m_{A}^{2}}{2} \int d^{3} x \mathbf{A}^{2}\right],
$$

where now $\nabla \cdot \mathbf{B}^{\mathrm{P}}(\mathbf{x})=(\pi / e)[\delta(\mathbf{x}-\mathbf{z})-\delta(\mathbf{x}-\overline{\mathbf{z}})]$. Also the divergence $\nabla \cdot\left(\nabla \times \mathbf{A}-\mathbf{B}^{\mathrm{P}}\right)$ is non-zero only at the location of the artificially introduced monopoles. In the absence of monopoles it follows that only the transverse part of $\mathrm{h}$ couples to $\nabla \times \mathbf{A}-\mathbf{B}^{\mathrm{P}}$. We therefore restrict the integral over the auxiliary field $h$ to the transverse degrees of freedom. This is justified by considering the field equation for $\mathrm{h}$ following from (65)

$$
\mathbf{h}=i\left(\nabla \times \mathbf{A}-\mathbf{B}^{\mathrm{P}}\right)=i \mathbf{h} .
$$

It tells us that apart from a factor $i$, the fluctuating field $\mathrm{h}$ may be thought of as representing the local induction $\mathbf{h}$, which we know to be divergence-free in the absence of monopoles.

The integral over the vector potential is again easily carried out by substituting the field equation for $\mathbf{A}$,

$$
\mathbf{A}=\frac{i}{m_{A}^{2}} \nabla \times \mathrm{h}
$$


back into (65), with the result

$$
\left\langle V_{\mathrm{r}}\left(L_{\mathbf{z}}\right) V_{\mathrm{r}}^{*}\left(L_{\overline{\mathbf{z}}}\right)\right\rangle=\int \mathcal{D} \mathrm{h} \delta(\nabla \cdot \mathrm{h}) \exp \left\{-\frac{1}{2} \int d^{3} x\left[\frac{1}{m_{A}^{2}}(\nabla \times \mathrm{h})^{2}+\mathrm{h}^{2}\right]-i \int d^{3} x \mathrm{~h} \cdot \mathbf{B}^{\mathrm{P}}\right\} .
$$

We have incorporated a $\delta$ function enforcing explicitly the constraint $\nabla \cdot \mathrm{h}=0$. In the present formulation this constraint is an intrinsic part of the description of a fluctuating massive vector field. For a non-fluctuating field it is a consequence of the field equation of h:

$$
-\frac{1}{m_{A}^{2}}\left(\partial_{i} \partial_{j}-\nabla^{2} \delta_{i j}\right) h_{j}-h_{i}-i B_{i}^{\mathrm{P}}=0
$$

Applying $\partial_{i}$ to this equation, we obtain $\nabla \cdot \mathrm{h}=0$ provided no monopoles are present and the mass $m_{A}$ is non-zero.

Expression (68) shows that the test tube described by the plastic field $\mathbf{B}^{\mathrm{P}}$ couples to the fluctuating massive vector field $\mathrm{h}$, with a coupling constant given by $g=(\pi / e) m_{A}=2 \pi w$ as in two dimensions. As $T$ approaches the critical temperature from below, $w$ goes to zero, and $\mathrm{h}$ decouples from the test tube described by $\mathbf{B}^{\mathrm{P}}$. After carrying out the integral over $\mathrm{h}$ in $(68)$ we recover the result (62).

The fact that the magnetic field has a finite penetration depth in the superconducting phase is reflected by the mass term of the $\mathrm{h}$ field.

It is interesting to consider the limit $m_{A} \rightarrow 0$ in detail, where the massive vector field decouples from the magnetic vortex. This limit yields the constraint $\nabla \times \mathrm{h}=0$ which can be solved by setting $\mathrm{h}=\nabla \gamma$. The correlation function $\left\langle V_{\mathrm{r}}\left(L_{\mathbf{z}}\right) V_{\mathrm{r}}^{*}\left(L_{\overline{\mathbf{z}}}\right)\right\rangle$ then takes the simple form

$$
\left\langle V_{\mathrm{r}}\left(L_{\mathbf{z}}\right) V_{\mathrm{r}}^{*}\left(L_{\overline{\mathbf{z}}}\right)\right\rangle=\int \mathcal{D} \gamma \exp \left[-\frac{1}{2} \int d^{3} x(\nabla \gamma)^{2}+i \int d^{3} x \gamma \rho\right]
$$

where $\rho$ is the monopole density. In the absence of monopoles, the theory reduces to that of a free massless mode $\gamma$ that may be thought of as representing the magnetic scalar potential. This follows from combining the physical interpretation of the vector field $h$ (66) with the equation $\mathrm{h}=\nabla \gamma$. Specifically, 


$$
\nabla \gamma=i\left(\nabla \times \mathbf{A}-\mathbf{B}^{\mathrm{P}}\right) .
$$

Using the definition of the monopole density, $\rho(\mathbf{x})=(\pi / e)[\delta(\mathbf{x}-\mathbf{z})-\delta(\mathbf{x}-\overline{\mathbf{z}})]$, we see that in terms of the field $\gamma$ the correlation function reads

$$
\left\langle V_{\mathrm{r}}\left(L_{\mathbf{z}}\right) V_{\mathrm{r}}^{*}\left(L_{\overline{\mathbf{z}}}\right)=\left\langle\mathrm{e}^{(\pi / e) i[\gamma(\mathbf{z})-\gamma(\overline{\mathbf{z}})]}\right\rangle .\right.
$$

This demonstrates that the operator $V_{\mathrm{r}}\left(L_{\mathbf{z}}\right)$ describing the test tube, which was introduced in (48) in a real-space formulation involving the singular plastic field $\mathbf{B}^{\mathrm{P}}$ (47), is now represented as an ordinary field. Since we are in the normal conducting phase, where $V_{\mathrm{r}}$ develops a non-zero expectation value, the presence of the phase $\gamma$ indicates that this expectation value breaks a global U(1) symmetry, with $\gamma$ the ensuing Goldstone field. This will be further clarified below.

Equation (72) reveals in addition that in the normal conducting phase the Dirac string looses its physical relevance, the right-hand side depending only on the end points $\mathbf{z}$ and $\overline{\mathbf{z}}$, not on the line $L_{\mathbf{z} \overline{\mathbf{z}}}$. This fact is also apparent from our starting formula (57), where the last term and therefore any reference to $L_{\mathbf{z}}$ disappears in the limit $m_{A} \rightarrow 0$. It makes no sense to talk about magnetic vortices in this phase because they are condensed and do not exist as physical excitations. There is also no non-trivial topology to assure their stability.

We are now in a position to derive the dual theory of a $3 D$ superconductor. This theory features a grand-canonical ensemble of fluctuating closed magnetic vortices, of arbitrary shape and length, which have a steric repulsion, i.e., a loop gas of magnetic vortices. We know that such an ensemble can be described by a disorder field theory, consisting of a complex $|\psi|^{4}$ theory. On the other hand, our study of a single magnetic vortex revealed that it couples with a coupling constant $g$ to the fluctuating vector field $h$. These two observations uniquely determine the dual theory in the London limit as being given by [25,26,8, 18, 19

$$
Z=\int \mathcal{D} \mathrm{h} \mathcal{D} \psi^{*} \mathcal{D} \psi \delta(\nabla \cdot \mathrm{h}) \exp \left(-H_{\psi}\right)
$$

with 


$$
H_{\psi}=\int d^{3} x\left[\frac{1}{2 m_{A}^{2}}(\nabla \times \mathrm{h})^{2}+\frac{1}{2} \mathrm{~h}^{2}+\left|\left(\nabla-i \frac{\pi}{e} \mathrm{~h}\right) \psi\right|^{2}+m_{\psi}^{2}|\psi|^{2}+u|\psi|^{4}\right],
$$

where the $\psi$ field is minimally coupled to the vector field h. Equation (74) replaces the lattice Hamiltonian (45) near the critical point. It is a description of the superconducting state in terms of physical variables: the field $\mathrm{h}$ describes the local induction, whereas $\psi$ accounts for the loop gas of magnetic vortices. There are no other physical objects present in a superconductor. The dual theory has no local gauge symmetry because the vector field $\mathrm{h}$ is massive. In fact, the two observations are connected. The presence of a local gauge symmetry in a given theory may be looked upon as reflecting a redundancy in the description. Since the dual theory is formulated in terms of physical variables, there is no redundancy, and thus no local gauge symmetry.

Although (73) was derived starting from the London limit, it is also relevant near the phase transition. The point is that integrating out the size fluctuations of the scalar field $\phi$ would only generate higher-order interaction terms and a possible change of the mass and interaction parameter $m_{\psi}$ and $u$. But these modifications do not alter the critical behavior of the theory.

The energy $M_{V}$ (61) appears in the dual theory as a one-loop on-shell mass correction stemming from the graph depicted in Fig. 2, which we now interpret as a Feynman graph. The straight and wiggly lines represent the $\psi$ and A field correlation functions, respectively.

A measure for the interaction strength of a massive vector field in $3 D$ is given by the dimensionless parameter equal to the square of the coupling constant multiplied by the range of the interaction. For the dual theory this factor is $g^{2} / m_{A} \sim m_{A} / e^{2}$, which is the inverse of the strength of the electromagnetic gauge field $\mathbf{A}$ in the superconducting phase. This is a common feature of theories which are dual to each other.

Another notable property of the dual theory is that in the limit $e \rightarrow 0$ it changes into a local gauge theory [8],

$$
H_{\psi} \rightarrow \int d^{3} x\left[\frac{1}{2}(\nabla \times \mathrm{h})^{2}+|(\nabla-i g \mathrm{~h}) \psi|^{2}+m_{\psi}^{2}|\psi|^{2}+u|\psi|^{4}\right],
$$

as can be checked by rescaling the dual field $h$ in the Hamiltonian (74). 
We next investigate what happens with the dual theory when we approach the critical temperature. Remember that $w$ and therefore $m_{A}$ tends to zero in the limit where $T$ tends to the critical temperature from below. From the first term in the Hamiltonian (74) it again follows that $\nabla \times \mathrm{h} \rightarrow 0$ in this limit, so that we can write once more $\mathrm{h}=\nabla \gamma$, and (74) becomes

$$
H_{\psi}=\int d^{3} x\left[\frac{1}{2}(\nabla \gamma)^{2}+\left|\left(\nabla-i \frac{\pi}{e} \nabla \gamma\right) \psi\right|^{2}+m_{\psi}^{2}|\psi|^{2}+u|\psi|^{4}\right] .
$$

This equation shows that $\gamma$, representing the magnetic scalar potential, cannot be distinguished from the phase of the disorder field. Indeed, let $(\pi / e) \vartheta$ be this phase. Then, the canonical transformation $\vartheta \rightarrow \vartheta+\gamma$ absorbs the scalar potential into the phase of $\psi$; the first term in (76) decouples from the theory and yields a trivial contribution to the partition function. In this way, the dual theory reduces to a pure $|\psi|^{4}$ theory

$$
H_{\psi}=\int d^{3} x\left(|\nabla \psi|^{2}+m_{\psi}^{2}|\psi|^{2}+u|\psi|^{4}\right) .
$$

It was already concluded that in the high-temperature phase the magnetic vortices proliferate as indicated by the fact that $\psi$, giving a field theoretic description of the loop gas of these objects, develops a non-zero expectation value at the transition point. This transition is triggered by a change in sign of $m_{\psi}^{2}$. In the London limit the Hamiltonian (77) then takes the simple form

$$
H_{\psi}=\int d^{3} x\left[\frac{1}{2} v^{2}\left(\frac{\pi}{e}\right)^{2}(\nabla \gamma)^{2}\right],
$$

with $v$ the expectation value of the disorder field, $v / \sqrt{2}=\langle|\psi|\rangle$, and where we now represented the phase of $\psi$ by $(\pi / e) \gamma$ to bring out the fact that $\gamma$ describes the magnetic scalar potential. As we will demonstrate below, $v$ has the value $v=e / \pi$ [9] of an inverse flux quantum, so that with our normalization choice of the phase of the $\psi$ field, Eq. (78) takes the canonical form.

The picture of the superconducting phase transition that emerges in the dual formulation of the Ginzburg-Landau theory is the following. When the critical temperature is approached 
from below, there is a proliferation of magnetic vortices. We recall that in the London limit parallel vortices repel each other, so that a single vortex prefers to crumple. Near $T_{\mathrm{c}}$ we then have a spaghetti of vortices which fill the space completely at and above the transition temperature. Since inside the core of a vortex one has the normal phase, the system thus becomes normal conducting. Whereas in the Ginzburg-Landau formulation a magnetic vortex is described by a singular plastic field $\mathbf{B}^{\mathrm{P}}$, in the dual formulation it is represented by

the Noether current $j_{i}=\psi^{*} \overleftrightarrow{\partial_{i}} \psi-2 i g \mathrm{~h}_{i} \psi^{*} \psi$. This follows from comparing the terms coupling linearly to the fluctuating $\mathrm{h}$ field. In the normal conducting phase the field $\psi$ develops a vacuum expectation value, and thereby breaks the global U(1) symmetry of the $|\psi|^{4}$ theory; $\gamma$ is the ensuing Goldstone field. The Noether current becomes in the London limit $\mathbf{j}=\nabla \gamma$, with $\gamma$ representing the massless photon of the high-temperature phase. It should be noted that at $T_{\mathrm{c}}$ the fluctuating local field $\mathrm{h}$ decouples from $\psi$ because $g \rightarrow 0$.

\section{THE MIXED STATE}

In the previous section it was shown that the dual theory of the Ginzburg-Landau model features a loop gas of magnetic vortices, i.e., closed random vortices of arbitrary shape and length which are generated by fluctuations. It is well known that magnetic vortices can also be generated by applying an external magnetic field $H$. Above a certain critical value $H_{\mathrm{c}_{1}}$ magnetic vortices start to penetrate the superconductor, provided it is a type-II superconductor. Below this lower critical field, the Meissner effect expels all flux lines from the system. In thermodynamic equilibrium the vortices in the so-called mixed state form a $2 D$ triangular lattice perpendicular to the applied field, a so-called Abrikosov flux lattice 233 (see Fig. 3). The $2 D$ vortex density in such a lattice is given by $\rho_{\otimes}=2 /\left(\sqrt{3} a^{2}\right)$, with $a$ the lattice spacing. The magnetic flux $\Phi=\int d^{2} x B$ through the lattice, which is given by the number of vortices $N$ multiplied by the fundamental flux unit $\pi / e$ carried by a single flux tube, increases with the applied field. At a second critical value $H_{\mathrm{c}_{2}}$ the magnetic induction $B$ becomes homogeneous and saturates the applied field; no more vortices are nucleated. 
The maximum vortex density $\rho_{\otimes, \max }$ reads

$$
\rho_{\otimes, \max }=H_{\mathrm{c}_{2}} \frac{e}{\pi}=\frac{1}{2 \pi \xi^{2}}
$$

where $H_{\mathrm{c}_{2}}=1 /\left(2 e \xi^{2}\right)$ is the upper critical field expressed in terms of the coherence length $\xi=1 /\left|m_{\phi}\right|$ [27]. At this value the magnetic vortices are closely packed and the system becomes normal conducting (see below). The area that can be assigned to a single vortex is $S_{\otimes}=1 / \rho_{\otimes}=2 \pi \xi^{2}$, so that $N S_{\otimes}$ covers the whole surface $S$ perpendicular to the applied field.

Let us study the Abrikosov flux lattice in the London limit, where the thickness of a magnetic vortex is considered to be infinitesimal small as compared to the penetration depth $\lambda=1 / m_{A}$. In this limit, where $\left|m_{\phi}\right|=1 / \xi$ may be taken to be infinite, the superconducting order field $\phi$ is frozen in, so that there is only one type of interaction between the magnetic vortices mediated by the gauge field A. This magnetic interaction is repulsive for two parallel vortices. (Outside the London limit, where the coherence length is finite, there is a second interaction mediated by $\phi$. This interaction is attractive for parallel vortices.) The Abrikosov flux lattice is easily understood in the London limit. Due to the repulsive magnetic interaction the vortices are driven apart, but this is called to an halt by the finiteness of the system. The vortices then order themselves in a regular lattice - which turns out to be a triangular lattice - so as to minimize the repulsive interaction.

The lattice is described by the plastic field $\mathbf{B}^{\text {ext }}$ which has only a component in say the negative third direction:

$$
B^{\mathrm{ext}}(\mathbf{x})=-\sum_{\alpha} \frac{\pi}{e} \int d x_{3} \delta\left(\mathbf{x}-\mathbf{x}_{\alpha}\right)
$$

where the sum is over all lattice points $\mathbf{x}_{\alpha}$, and where we took the external field as pointing in the third direction. In a first approximation, the $\psi$ field in $(\sqrt{74})$ may be neglected. The field equation for the local field $\mathbf{h}=(0,0, h(\mathbf{x}))$ which we infer from (74) augmented with the term $-i \mathrm{~h} \cdot \mathbf{B}^{\text {ext }}$ then reads

$$
-\lambda^{2} \nabla^{2} h+h=-B^{\mathrm{ext}}
$$


where we accounted for the factor $i$ between the fluctuating field $\mathrm{h}$ and the physical local field $\mathbf{h}$. The form (81) is well-known, and can also be obtained from the Ginzburg-Landau theory. We consider the lattice in two different limits. The first limit is the one of low vortex density. Just above the lower critical field $H_{\mathrm{c}_{1}}$ the density is such that one may consider the system as non-interacting. In this case the field equation (81) has the solution

$$
h(\mathbf{x})=\frac{1}{2 e \lambda^{2}} \sum_{\alpha} K_{0}\left(\lambda^{-1}\left|\mathbf{x}-\mathbf{x}_{\alpha}\right|\right)
$$

where $K_{0}$ is a modified Bessel function. The corresponding free energy (density) is

$$
\begin{aligned}
F & =-\frac{1}{2 V} \int d^{3} x h B^{\mathrm{ext}} \\
& =\frac{\pi}{4 e^{2} \lambda^{2} S} \sum_{\alpha, \beta} K_{0}\left(\lambda^{-1}\left|\mathbf{x}_{\alpha}-\mathbf{x}_{\beta}\right|\right)
\end{aligned}
$$

with $V$ the volume of the system, and $S$ the area perpendicular to the applied field. In the limit of low density one only has to account for the self-interaction $(\alpha=\beta)$ and the nearest-neighbor interaction, so that the free energy can be approximated by

$$
F=B H_{\mathrm{c}_{1}}\left[1+\frac{6}{\ln (\kappa)} K_{0}\left(\lambda^{-1} a\right)\right],
$$

where we used the standard result that $H_{\mathrm{c}_{1}}=\ln (\kappa) /\left(4 e \lambda^{2}\right)$ [27]. This equation shows that by raising the applied field an increasing part of the field energy is used to overcome the repulsive interaction between the magnetic vortices. This is represented by the last term in (84), which not only contains a factor of $B$, like the first term, but in addition depends on the lattice spacing $a$. For increasing fields $a$ decreases, implying that indeed the nearest-neighbor interaction term becomes more important. This observation can be nicely illustrated by solving the thermodynamic relation $H=\partial F / \partial B$ in terms of the magnetic induction $B$, which is related to the vortex density via $B=(\pi / e) \rho_{\otimes}$ (see Fig. 屯). For fields slightly larger than $H_{\mathrm{c}_{1}}$, there is a rapid increase in the vortex density which for larger fields, when the interaction between vortices becomes important, is leveled off.

This indicates that the existence of a mixed state is due to the repulsive magnetic interaction between magnetic vortices. If one takes away the interaction term in the free energy, 
one finds a magnetization curve resembling that of type-I superconductors, which do not support a mixed state. The picture is confirmed by the fact that the two critical field values $H_{\mathrm{c}_{1}}$ and $H_{\mathrm{c}_{2}}$, marking the boundaries of the mixed state, become closer to each other the smaller the value of the Ginzburg-Landau parameter $\kappa=\lambda / \xi$ is. With $\xi$ kept fixed, a smaller $\kappa$ value implies a smaller penetration depth which results in a stronger shielding of the repulsive magnetic force.

There exist dirty superconductors which have a Ginzburg-Landau parameter close to the critical value $\kappa=1 / \sqrt{2}$ separating a type-II from a type-I superconductor. Such a value indicates that the screening of the two forces which are present outside the London limit, are of the same order of magnitude. These so-called type-II/1 materials exhibit a remarkable experimental phenomenon. If the lower critical field $H_{\mathrm{c}_{1}}$ is crossed from below, a whole flux lattice jumps in instead of single vortices being produced one by one as is the case of a deep type-II superconductor. This is related to the fact that for these materials the dominant force at large distances is the attractive one mediated by the superconducting order field [28], while at short distances the repulsive magnetic interaction dominates (see Fig. (5). The lattice spacing in the flux lattice produced in such a type-II/1 superconductor is namely an equilibrium spacing resulting from the balance between the repulsive and attractive force. It is indicated by $a_{0}$ in Fig. 5 .

Type-I superconductors, for which the two critical fields $H_{\mathrm{c}_{1}}$ and $H_{\mathrm{c}_{2}}$ have the same value $H_{\mathrm{c}}$, and for which $\kappa<1 / \sqrt{2}$, do not support a mixed state because the screening length of the repulsive magnetic force for those materials is smaller than the coherence length $\xi$ defining the core radius. That is, effectively the magnetic repulsion is screened to zero outside the vortex core, so that vortices in a type-I material experience only an attractive force. They will consequently coalesce, with the result that the entire sample becomes normal.

The other limit we briefly discuss is that of high-vortex density, where the lattice spacing $a$ is much smaller than the penetration depth. Because of the periodicity of the lattice, one can consider a single unit cell and the Fourier transform becomes a series 


$$
h(\mathbf{x})=\sum_{\{\mathbf{l}\}} h\left(\mathbf{k}_{l}\right) e^{i \mathbf{k}_{l} \cdot \mathbf{x}},
$$

where $\mathbf{l}$ stands for the index pair $\mathbf{l}=\left(l_{1}, l_{2}\right)$, and $\mathbf{k}_{l}$ is the reciprocal lattice vector

$$
\mathbf{k}_{l}=\frac{4 \pi}{\sqrt{3} a}\left(l_{1} \mathbf{E}^{1}+l_{2} \mathbf{E}^{2}\right)
$$

with $\mathbf{E}^{1}=\left(\frac{\sqrt{3}}{2},-\frac{1}{2}\right)$ and $\mathbf{E}^{2}=(0,1)$ two vectors spanning the reciprocal lattice. The Fourier components of the plastic field $B^{\text {ext }}(\mathbf{x})$ are

$$
B^{\mathrm{ext}}\left(\mathbf{k}_{l}\right)=-\frac{\pi}{e} \rho_{\otimes} \mathrm{e}^{i \mathbf{k}_{l} \cdot \mathbf{x}_{\alpha}}
$$

with $\mathbf{x}_{\alpha}$ the position of the vortex we are considering. This leads to the free energy

$$
F=\frac{1}{2}\left(\frac{\pi}{e}\right)^{2} \rho_{\otimes}^{2} \sum_{\{1\}} \frac{1}{1+\lambda^{2} \mathbf{k}_{l}^{2}} .
$$

In deriving this we used the fact that all vortices give the same contribution, so that it suffice to consider a single vortex, say, located at the origin $\mathbf{x}_{\alpha}=0$. The term with $\mathbf{l}=0$ in (88) represents the homogeneous part. The remaining sum is diverging for large $\mathbf{l}$, but this is artificial, stemming from the fact that we are in the London limit where the core radius is taken to be infinitesimal small. As before, we take an ultraviolet cutoff of the order of the inverse coherence length $\left|m_{\phi}\right|=1 / \xi$. Since in the limit of large vortex density $\lambda / a>>1$, one can for $\mathbf{l} \neq 0$ neglect 1 compared to $\lambda^{2} \mathbf{k}_{l}^{2}$ in the denominator of (88). In this way, approximating the sum by an integral, one finds for the free energy

$$
\begin{aligned}
F & =\frac{B^{2}}{2}+\frac{1}{2}\left(\frac{\pi}{e}\right)^{2} \rho_{\otimes} \int_{k_{\min }<|\mathbf{k}|<k_{\max }} \frac{d^{2} k}{(2 \pi)^{2}} \frac{1}{(\lambda \mathbf{k})^{2}} \\
& =\frac{B^{2}}{2}+\frac{1}{2 \kappa^{2}} B H_{\mathrm{c}_{2}} \ln \left(\frac{k_{\max }}{k_{\min }}\right),
\end{aligned}
$$

The infrared cutoff is taken of the order of the inverse lattice spacing $k_{\min } \sim 1 / a$. To be precise,

$$
\frac{k_{\max }}{k_{\min }}=\beta \frac{a}{\xi}=\beta \frac{\sqrt{4 \pi}}{3^{1 / 4}} \sqrt{\frac{H_{\mathrm{c}_{2}}}{B}} \approx 1.026 \sqrt{\frac{H_{\mathrm{c}_{2}}}{B}},
$$

where $\beta \approx 0.381$ is a numerical factor [29] which is adjusted so that the approximate calculation is in accordance with the exact result. One thus finds for the free energy 


$$
F \approx \frac{B^{2}}{2}-\frac{1}{4 \kappa^{2}} B H_{\mathrm{c}_{2}} \ln \left(\frac{B}{H_{\mathrm{c}_{2}}}\right)
$$

which in the limit $B \rightarrow H_{\mathrm{c}_{2}}$ reduces to the free energy of the normal phase, as it should.

Intuitively, the phase transition at $H_{\mathrm{c} 2}$ may be pictured as follows. When increasing the external field, the Abrikosov flux lattice becomes denser, and the spherical cross-section of the normal-conducting cores will be continuously deformed into a hexagonal one. This picture is nicely confirmed by numerical calculations [30] (see Fig. 6). Precisely at $H_{\mathrm{c}_{2}}$ the magnetic vortices are densely packed, and the superconducting cell borders are squeezed to zero thickness. In this way the mixed state becomes a homogeneous, normal conducting state.

\section{THE NORMAL PHASE}

In Sec. $\mathrm{Z}$ we saw that the normal conducting phase is described by a disorder theory, consisting of a $|\psi|^{4}$ theory in the broken-symmetry phase. This theory possesses again topological defects, viz., vortices, known from the theory of superfluid ${ }^{4} \mathrm{He}$ [31]. In this section a dual formulation of the $|\psi|^{4}$ theory is considered in which the grand canonical ensemble of closed vortices is described by a field theory. The theory turns out to be the original Ginzburg-Landau model (in the high-temperature phase). This is not surprising because a dual map of a dual theory should recover the original model.

We proceed in the same manner as in Sec. $\nabla$ and consider a vortex ending at the point $\mathbf{z}$ inside the system. To describe such an object we would introduce in the London limit (78) of the $|\psi|^{4}$ theory the operator

$$
W\left(L_{\mathbf{z}}\right)=\mathrm{e}^{-\frac{1}{2} v^{2} \int d^{3} x\left(\boldsymbol{\theta}^{\mathrm{P}}\right)^{2}} \exp \left(\frac{\pi}{e} v^{2} \int d^{3} x \nabla \gamma \cdot \boldsymbol{\theta}^{\mathrm{P}}\right)
$$

where the first factor is a singular contribution due to the core of the vortex, cf. (48). In (92), the factor $\pi / e$ stems from our normalization choice of the phase of the $\psi$ field, and the vector field $\boldsymbol{\theta}^{\mathrm{P}}$, first introduced in the context of superfluid ${ }^{4} \mathrm{He}$ [8], should be such that 
$\nabla \times \boldsymbol{\theta}^{\mathrm{P}}$ yields a delta function along the line $L_{\mathbf{z}}$ which starts at the point $\mathbf{z}$ and runs to infinity along the trajectory $\mathbf{y}(s)$ :

$$
\left(\nabla \times \boldsymbol{\theta}^{\mathrm{P}}\right)_{i}(\mathbf{x})=2 \pi \int_{L_{\mathbf{z}}} d s \frac{d y_{i}}{d s} \delta[\mathbf{x}-\mathbf{y}(s)]
$$

with the divergence of this equation yielding a delta function at the point $\mathbf{z}$. But this is impossible since for a regular field $\nabla \cdot\left(\nabla \times \boldsymbol{\theta}^{\mathrm{P}}\right)=0$. Hence, the only regular $\boldsymbol{\theta}^{\mathrm{P}}$ field that can be constructed is one representing a closed, or infinitely long vortex $L$. To understand this in another way [8], imagine a sphere $\Sigma$ surrounding the hypothetical endpoint of a vortex at $\mathbf{z}$, with a little hole $\partial \Sigma$ where the vortex pierces the surface. While on the one hand the loop integral $\oint_{\partial \Sigma} d x_{i} \partial_{i} \gamma$ gives the vortex strength $2 \pi$, the surface integral $\int_{\Sigma} d^{2} x_{i} \epsilon_{i j k} \partial_{i} \partial_{j} \gamma$, on the other hand, vanishes since $\gamma$ is regular on $\Sigma$. This contradicts Stokes' theorem which states that both integrals should be equal and leads to the conclusion that vortices in a $|\psi|^{4}$ theory can only form finite closed loops, or infinite loops which are so to speak "closed at infinity".

It should be noted that the above argument does not apply to the Ginzburg-Landau theory. A magnetic vortex could start in a given point by simply introducing a magnetic monopole there. When described with help of a gauge potential, the monopole is inevitably accompanied by a Dirac string. Choosing the Dirac string to pierce the surface $\Sigma$ surrounding the monopole at $\partial \Sigma$, one obtains $\oint_{\partial \Sigma} d x_{i} A_{i}=\int_{\Sigma} d^{2} x_{i} \epsilon_{i j k} \partial_{j} A_{k}$, where the right-hand side measures the magnetic flux through the surface $\Sigma$ (see Fig. 7). Both sides of the equation yield the same result, in accordance with Stokes' theorem.

For a closed loop $L$, the expectation value of $W(L)$ is given by

$$
\langle W(L)\rangle=\int \mathcal{D} \gamma \exp \left[-\frac{1}{2} v^{2} \int d^{3} x\left(\frac{\pi}{e} \nabla \gamma-\boldsymbol{\theta}^{\mathrm{P}}\right)^{2}\right] .
$$

The integration over $\gamma$ can be carried out by substituting the field equation of the Goldstone field

$$
\gamma(\mathbf{x})=-\frac{e}{\pi} \int d^{3} y \Delta^{0}(\mathbf{x}-\mathbf{y}) \nabla \cdot \boldsymbol{\theta}^{\mathrm{P}}(\mathbf{y})
$$


where $\Delta^{0}$ is the correlation function (53) with $m_{A}=0$. This yields an expression

$$
\langle W(L)\rangle=\exp \left\{-\frac{1}{2} v^{2} \int d^{3} x \int d^{3} y\left[\nabla \times \boldsymbol{\theta}^{\mathrm{P}}(\mathbf{x})\right]_{i} \Delta^{0}(\mathbf{x}-\mathbf{y})\left[\nabla \times \boldsymbol{\theta}^{\mathrm{P}}(\mathbf{y})\right]_{i}\right\},
$$

very similar to the one obtained for a magnetic vortex in the superconducting phase, Eq. (57). Using (93), we obtain, cf. (60)

$$
\langle W(L)\rangle=\mathrm{e}^{-M_{W}|L|},
$$

with $|L|$ the vortex length and, cf. (61)

$$
M_{W}=\frac{\pi v^{2}}{2} \ln \left(\frac{\left|m_{\psi}\right|^{2}}{\mu^{2}}\right)
$$

its energy per unit length. In analogy with the previous calculation, we have taken the mass $\left|m_{\psi}\right|$ of the scalar field appearing in the theory as ultraviolet cutoff. Since $\gamma$ is massless, $M_{W}$ diverges in the infrared. This is regularized by introducing a small mass $\mu$.

In order to find the dual theory which gives a field theoretic description of the vortex loop gas, we rewrite the expectation value $\langle W(L)\rangle$ via a Hubbard-Stratonovich transformation as

$$
\langle W(L)\rangle=\int \mathcal{D} \gamma \mathcal{D} \mathbf{b} \exp \left\{-\int d^{3} x\left[\frac{1}{2} \mathbf{b}^{2}+i v \mathbf{b} \cdot\left(\frac{\pi}{e} \nabla \gamma-\boldsymbol{\theta}^{\mathrm{P}}\right)\right]\right\}
$$

The integral over the $\gamma$ fluctuations now yields the constraint $\nabla \cdot \mathbf{b}=0$, demanding $\mathbf{b}$ to be the rotation of a vector field, $\mathbf{b}=\nabla \times \mathbf{A}$. This gives

$$
\langle W(L)\rangle=\int \mathcal{D} \mathbf{A} \exp \left\{-\int d^{3} x\left[\frac{1}{2}(\nabla \times \mathbf{A})^{2}-2 \pi i v \mathbf{A} \cdot \mathbf{J}\right]\right\},
$$

where $J_{i}(\mathbf{x}):=\oint_{L} d y_{i} \delta(\mathbf{x}-\mathbf{y})$ describes the closed vortex. It is natural to interpret the fluctuating massless gauge field $\mathbf{A}$ as the electromagnetic gauge field. This identification yields the relation we alluded to above

$$
2 \pi v=2 e
$$

between the expectation value $v$ of the disorder field $\psi$ and the electric charge $2 e$. It is the analog of relation (19) between the expectation value $w$ of the superconducting order field $\phi$ and the coupling constant $g=(\pi / e) m_{A}$ of the dual theory: 


$$
2 \pi w=g
$$

The expectation value (100) we now recognize as the Wilson loop. Since $v$ vanishes as $T$ approaches the critical temperature from above, the coupling constant vanishes at the critical point and A decouples from the vortex. Precisely the same phenomenon happened with the magnetic vortex in the superconducting phase. Adding a gauge-fixing term to (100) and carrying out the integration over the gauge field, we obtain

$$
\langle W(L)\rangle=\left\langle\mathrm{e}^{2 i e \oint_{L} d \bar{y}_{i} A_{i}}\right\rangle=\exp \left[-2 e^{2} \int d^{3} x d^{3} y J_{i}(\mathbf{x}) \Delta_{i j}^{0}(\mathbf{x}-\mathbf{y}) J_{j}(\mathbf{y})\right],
$$

where $\Delta_{i j}^{0}$ is the correlation function (51) with $m_{A}=0$. Because we consider closed vortices, for which $\nabla \cdot \mathbf{J}=0$, only the first term of the correlation function contributes, so that the result is independent of gauge choice and is given by the previous expression (96).

If we consider a loop gas of vortices, we recover, following the same line of arguments as in Sec. V, the (normal phase of the) original Ginzburg-Landau model (11). As expected, the dual map of the dual theory gives back the original model.

Since the expectation value $v$ of the $\psi$ field vanishes when the critical point is approached from above, it follows that also the energy (98) tends to zero here. This supports the picture that the phase transition in a pure $|\psi|^{4}$ theory is associated with the proliferation of vortices 8.

The question arises now How can we physically understand a vortex loop in the normal phase? As is well known from ${ }^{4}$ He physics [31,8], inside the core of a ${ }^{4}$ He vortex, the superfluid order parameter vanishes, indicating that the core consists of normal fluid. Translated into the present context, where a finite expectation value of the disorder $\psi$ field indicates the onset of the normal conducting phase, we are to interpret its vanishing inside the vortex core as the absence of the normal phase, i.e., as the presence of the superconducting phase. If one takes the Maxwell equation

$$
\nabla \times \mathbf{h}=2 e \mathbf{j}
$$


as the defining equation of the electromagnetic current $\mathbf{j}$, one has to view the purely imaginary object $2 \pi i v \mathbf{J}$ in (100) as describing an electric circuit. (The analog between vortices and electric currents was first pointed out by von Helmholtz.) At first glance the presence of the factor $i$ seems strange. In particular, the Biot-Savart law for these currents yields the opposite sign from what is usually the case: we find two parallel currents repelling instead of attracting each other because they carry an imaginary charge. (This can be checked by considering the interaction energy $E_{\text {int }}$ between two parallel vortices

$$
E_{\mathrm{int}}=2 \pi^{2} v^{2} \int d x_{i} \int d y_{i} \frac{1}{|\mathbf{x}-\mathbf{y}|},
$$

which is positive.) But a closer inspection reveals that this has to be the case. Remember that an electric circuit generates a magnetic moment

$$
\mathbf{m}=e \int d^{3} x(\mathbf{x} \times \mathbf{j})
$$

orthogonal to the surface enclosed by the loop. (Note that we defined the current without a charge factor - $2 e$ in our case - included, that is why the equation for $\mathbf{m}$ contains a factor $\frac{1}{2} 2 e=e$ ). If we take two real-life circuits I and II as sketched in Fig. 8, where the lower laying loop I is held fixed while the upper one is free to rotate, then the latter would settle such that the two magnetic moments point in the same direction. A state where these real-life electric circuits are condensed would inevitably be connected with a permanent magnetization. Due to the fact that vortex loops carry an imaginary charge, there is an antiferromagnetic coupling rather than a ferromagnetic one between the loops, and a vortex condensate has zero magnetization.

We note that because of the imaginary charge, the local field generated by a vortex loop $L$ is also purely imaginary as follows from Ampere's law,

$$
\mathbf{h}(\mathbf{x})=i \frac{v}{2} \oint d \mathbf{y} \times \frac{\mathbf{x}-\mathbf{y}}{|\mathbf{x}-\mathbf{y}|^{3}}=i \frac{v}{2} \nabla \Omega(\mathbf{x}),
$$

where $\Omega$ is the solid angle that the loop subtends at $\mathbf{x}$ (see Fig. 9). The same result can be obtained from the dual theory, bearing in mind that the local field, apart from a factor $i$, can be identified with the gradient of the phase variable $\gamma$ 


$$
\mathbf{h}=-i \nabla \gamma
$$

see (71). Rewriting the field equation (95) for $\gamma$, we find that this field can be related to the solid angle in the following way [8]

$$
\gamma(\mathbf{x})=\frac{e}{2 \pi} \int_{S} d^{2} y_{i} \frac{(\mathbf{x}-\mathbf{y})_{i}}{|\mathbf{x}-\mathbf{y}|^{3}}=-\frac{e}{2 \pi} \Omega
$$

where $d^{2} y_{i}$ is an element of the surface $S$ spanned by the loop. Together with (108) this yields the previous result (107). [The magnetic moment density, or magnetization, is represented in the dual theory by $\boldsymbol{\theta}^{\mathrm{P}}$. This follows from the fact that according to (94) a closed vortex couples to the magnetic field $\nabla \gamma$ via $\boldsymbol{\theta}^{\mathrm{P}}$.] The order parameter $V_{\mathrm{r}}(\mathbf{x})$ of the normal state essentially measures the angle $\Omega$

$$
V_{\mathrm{r}}(\mathbf{x})=\mathrm{e}^{i \pi \gamma(\mathbf{x}) / e}=\mathrm{e}^{i \Omega(\mathbf{x}) / 2}
$$

We recall that the operator $V(\mathbf{x})$ was constructed by putting a magnetic monopole at $\mathbf{x}$. With this kept in mind, Eq. (110) becomes obvious: $\Omega / 2$ is the magnetic flux through the closed vortex $L$ emanated by the monopole. As a last remark we note that one can chose two topologically different surfaces spanning the loop L (see Fig. 10). Both lead, however, to the same phase factor because $\Omega$ differs only by a factor of $4 \pi$.

\section{SUPERCONDUCTING ORDER PARAMETER}

In the previous section it was argued that the transition to the superconducting phase could be understood as a proliferation of (closed) vortices of the pure $|\psi|^{4}$ theory. This was concluded from the behavior of the real-space representation (94) of the single loop operator $W(L)$, which was shown to develop an expectation value when $T$ approaches the critical temperature from above. The question naturally arises Is $W(L)$ related to the superconducting order field $\phi$ ? To answer this question we have to investigate how $W(L)$ is described in terms of the variables of the Ginzburg-Landau theory. To this end we study the object 


$$
O\left(L_{\mathbf{z}}\right)=\mathrm{e}^{i \theta(\mathbf{z})} \mathrm{e}^{2 i e \int d^{3} x \mathbf{A}(\mathbf{x}) \cdot \mathbf{E}^{\mathrm{P}}(\mathbf{x})}
$$

where $\theta$ is the phase of $\phi$, the plastic field $\mathbf{E}^{\mathrm{P}}$ describes a static charge $2 e$ at $\mathbf{z}$,

$$
\nabla \cdot \mathbf{E}^{\mathrm{P}}(\mathbf{x})=\delta(\mathbf{x}-\mathbf{z})
$$

and $L_{\mathbf{z}}$ is a line emanating from $\mathbf{z}$ and running to infinity,

$$
E_{i}^{\mathrm{P}}=\int_{L_{\mathbf{z}}} d y_{i} \delta(\mathbf{x}-\mathbf{y})
$$

The second factor in (111) is incorporated in order to render the operator gauge invariant. Indeed, under a gauge transformation

$$
\mathbf{A}(\mathbf{x}) \rightarrow \mathbf{A}(\mathbf{x})+\nabla \Lambda(\mathbf{x}), \quad \theta(\mathbf{z}) \rightarrow \theta(\mathbf{z})+2 e \Lambda(\mathbf{z})
$$

so that

$$
O\left(L_{\mathbf{z}}\right) \rightarrow O\left(L_{\mathbf{z}}\right) \exp \left[2 i e \Lambda(\mathbf{z})+2 i e \int d^{3} x \nabla \Lambda(\mathbf{x}) \cdot \mathbf{E}^{\mathrm{P}}(\mathbf{x})\right]=O\left(L_{\mathbf{z}}\right)
$$

where in the last step we performed an integration by parts. To bring out the gauge invariance of $O\left(L_{\mathbf{z}}\right)$ more clearly we write it in the equivalent form

$$
O\left(L_{\mathbf{z}}\right)=\exp \left[-i \int d^{3} x(\nabla \theta-2 e \mathbf{A}) \cdot \mathbf{E}^{\mathrm{P}}\right]
$$

We will be working in the low-temperature phase of the Ginzburg-Landau model, where the gauge field is massive. We are interested in the expectation value

$$
\left\langle O\left(L_{\mathbf{z}}\right)\right\rangle=\int \mathcal{D} \mathbf{A} \mathcal{D} \theta O\left(L_{\mathbf{z}}\right) \mathrm{e}^{-H}
$$

with $H$ the Ginzburg-Landau Hamiltonian (2) in the London limit. Since both integrations are Gaussian, they are easily carried out to yield

$$
\left\langle O\left(L_{\mathbf{z}}\right)\right\rangle=\exp \left\{-\frac{1}{2} \int d^{3} x d^{3} y\left[\frac{1}{w^{2}} n(\mathbf{x}) \Delta(\mathbf{x}-\mathbf{y}) n(\mathbf{y})+(2 e)^{2} E_{i}^{\mathrm{P}}(\mathbf{x}) \Delta(\mathbf{x}-\mathbf{y}) E_{i}^{\mathrm{P}}(\mathbf{y})\right]\right\},
$$

with $n(\mathbf{x})=\delta(\mathbf{x}-\mathbf{z})$ the charge density. This expression closely resembles the one we found for the operator $V$ in (57), describing a magnetic vortex, which can be rewritten as 


$$
\left\langle V\left(L_{\mathbf{z}}\right)\right\rangle=\exp \left\{-\frac{1}{2} \int d^{3} x d^{3} y\left[\frac{1}{v^{2}} n(\mathbf{x}) \Delta(\mathbf{x}-\mathbf{y}) n(\mathbf{y})+g^{2} \bar{B}_{i}^{\mathrm{P}}(\mathbf{x}) \Delta(\mathbf{x}-\mathbf{y}) \bar{B}_{i}^{\mathrm{P}}(\mathbf{y})\right]\right\}
$$

where $\bar{B}_{i}^{\mathrm{P}}$ is defined so that it contains no factor $\pi / e, B_{i}^{\mathrm{P}}=(\pi / e) \bar{B}_{i}^{\mathrm{P}}$. We see that (118) can be obtained from (119) by simply replacing the high-temperature expectation value $v$ of the $\psi$ field by the low-temperature expectation value $w$ of the $\phi$ field, and by replacing the "magnetic" coupling $g$ by the electric coupling $2 e$. In this sense the operators $O$ and $V$ are dual to each other.

We continue to discuss the behavior of the expectation value (118) in the two phases. In the high-temperature phase we argued that there can only be closed vortices. This we achieve by setting $n(\mathbf{x})$ to zero in (118), so that only the last term survives. In fact, using the relation $\pi v=e$, we recover the right-hand side of (96). That is, the operators $O(L)$ and $W(L)$ are the same in the high-temperature phase:

$$
\langle O(L)\rangle=\langle W(L)\rangle=\mathrm{e}^{-M_{W}|L|}
$$

with $|L|$ the length of the vortex loop and $M_{W}$ given in (61).

In the low-temperature phase the (electric) vortices are condensed and for that reason not existing as physical excitations, only the endpoints are physical. The plastic field $\mathbf{E}^{\mathrm{P}}$ in (118) can then be written as a gradient of a potential $U^{\mathrm{P}}$,

$$
\mathbf{E}^{\mathrm{P}}=-\nabla U^{\mathrm{P}}
$$

with $\nabla^{2} U^{\mathrm{P}}(\mathbf{x})=-n(\mathbf{x})$. Taking a positive charge $2 e$ at $\mathbf{z}$ and a negative one $-2 e$ at $\overline{\mathbf{z}}$, we obtain for the correlation function

$$
\left\langle O\left(L_{\mathbf{z}}\right) O^{*}\left(L_{\overline{\mathbf{z}}}\right)\right\rangle=\exp \left[-\frac{1}{2 w^{2}} \int d^{3} x d^{3} y n(\mathbf{x}) \Delta^{0}(\mathbf{x}-\mathbf{y}) n(\mathbf{y})\right]
$$

where now $n(\mathbf{x})=\delta(\mathbf{x}-\mathbf{z})-\delta(\mathbf{x}-\overline{\mathbf{z}})$, and $\Delta^{0}(\mathbf{x}-\mathbf{y})$ is the massless scalar correlation function. For $\mathbf{x}=\mathbf{y}$ we have again a diverging self-interaction which is irrelevant and can be eliminated by defining a renormalized operator $O_{\mathrm{r}}$ in the same way as we did before in (59). We then find 


$$
\left\langle O_{\mathrm{r}}(\mathbf{z}) O_{\mathrm{r}}^{*}(\overline{\mathbf{z}})\right\rangle=\exp \left(\frac{1}{4 \pi w^{2}} \frac{1}{\left|L_{\mathbf{z} \overline{\mathbf{z}}}\right|}\right) .
$$

This low-temperature expression is completely analogous to the one in the high-temperature phase for the correlation function $\left\langle V_{\mathrm{r}}(\mathbf{z}) V_{\mathrm{r}}^{*}(\overline{\mathbf{z}})\right\rangle$, Eq. (62). Using the relation $e=\pi v$, we can write the latter as

$$
\left\langle V_{\mathrm{r}}(\mathbf{z}) V_{\mathrm{r}}^{*}(\overline{\mathbf{z}})\right\rangle=\exp \left(\frac{1}{4 \pi v^{2}} \frac{1}{\left|L_{\mathbf{z} \overline{\mathbf{z}}}\right|}\right) .
$$

For large separation $\left\langle O_{\mathrm{r}}(\mathbf{z}) O_{\mathrm{r}}^{*}(\overline{\mathbf{z}})\right\rangle \rightarrow 1$, implying that $O_{\mathrm{r}}(\mathbf{z})$ develops an expectation value in the superconducting phase. That is, $O_{\mathrm{r}}(\mathbf{z})$ is the superconducting order parameter. Being gauge invariant this operator makes no statement about the local U(1) symmetry. Referring back to the first representation (111) of the superconducting order parameter, we find that a non-zero expectation value indicates that the global $\mathrm{U}(1)$ symmetry parameterized by a constant transformation parameter $\Lambda_{0}$ is spontaneously broken.

In closing this section, we remark that the superconducting order parameter can also be represented in the dual theory. The result is that $\mathbf{E}^{\mathrm{P}}$ appears in (74) in the combination $\nabla \times \mathrm{h}-2 e \mathbf{E}^{\mathrm{P}}$ with the fluctuating $\mathrm{h}$ field. To derive this result it is prudent not to proceed in the manner we exploited before to obtain the dual theory (74) and linearize the gauge-field fluctuations, but instead linearize the $\theta$ fluctuations. The result can also be inferred using a duality argument, remembering that the disorder parameter was incorporated in the original theory by the combination $\nabla \times \mathbf{A}-\mathbf{B}^{\mathrm{P}}$, see Eq. (46).

\section{DISORDER FIELD THEORY FOR THE SUPERCONDUCTING PHASE TRANSITION}

We have argued that the dual description of the Ginzburg-Landau model is one in terms of physical variables, and that it possesses no local gauge symmetry. A disorder field $\psi$ was identified which vanishes in the superconducting phase, and which develops an expectation value in the normal conducting phase, thereby breaking a global $\mathrm{U}(1)$ symmetry. We recall that central to Landau's theory of continuous phase transitions is the presence of an order 
parameter which signals through its vacuum expectation value whether or not a certain symmetry is broken. This is precisely what the disorder field $\psi$ does. On that ground $\psi$ is ideally suited to formulate a Landau type of description of the superconducting phase transition [9:18]. To understand why the Ginzburg-Landau theory itself is not well suited to do this, we note that it has a local gauge symmetry. According to Elitzur's theorem [10] such a symmetry can never be broken, so that for a local symmetry no order parameter exists in the sense of Landau. This may be one of the reasons for the fact that no infrared stable fixed point was found within the Ginzburg-Landau formulation of the superconducting phase transition [32,33], although it is generally accepted that the transition is of second order in the type-II regime, and thus should possess such a point.

Below the transition temperature we saw that the dual theory consists of a $|\psi|^{4}$ theory coupled to a massive vector field. Above $T_{\mathrm{c}}$ this field decouples, and the disorder field $\psi$ simultaneously develops a vacuum expectation value. Despite the apparent difference in the description of the low- and high-temperature phase, it is readily argued that at the meanfield level the critical behavior is governed by a simple $|\psi|^{4}$ theory. This can be seen by integrating out the massive vector field in the low-temperature phase. Apart from irrelevant terms, this leads to only a change in the coefficients of the $|\psi|^{4}$ theory, no additional relevant terms such as $|\psi|^{3}$ are generated. Explicitly,

$$
H_{\psi, \text { eff }}=\int d^{3} x\left[|\nabla \psi|^{2}+\left(m_{\psi}^{2}-g^{2} \frac{m_{A}}{2 \pi}\right)|\psi|^{2}+\left(u-\frac{g^{4}}{4 \pi m_{A}}\right)|\psi|^{4}\right] .
$$

In deriving this effective Hamiltonian we used dimensional regularization; (irrelevant) higherorder terms were omitted. We note that all contributions stemming from the vector field $\mathrm{h}$ vanish in the limit $T$ approaching $T_{\mathrm{c}}$ from below, so that $H_{\psi, \text { eff }}$ reduces to (77) in this limit. It is well known that a $|\psi|^{4}$ theory with a positive coupling has a non-trivial infrared stable fixed point and undergoes a second-order phase transition. We therefore conclude that at the mean-field level also the superconducting phase transition is of second order if the system is sufficiently deep in the type-II regime. The same conclusion can be reached starting from the Ginzburg-Landau formulation. 
Below we will apply renormalization group theory to see if this conclusion holds also beyond mean-field theory. Halperin, Lubensky, and Ma [32] performed this study within the Ginzburg-Landau theory. Using an $\epsilon$ expansion, they showed that at the one-loop level the theory no longer possesses an infrared stable fixed point. They interpreted this as indicating that the transition is of first order. This conclusion was in accordance with results obtained by Coleman and Weinberg [34] who studied the electrodynamics of massless scalar mesons in four dimensions and discovered that at the one-loop level the photon acquires a mass. A study of the effective action shows a precocious onset of the Higgs mechanism with a sudden appearance of a finite photon mass. This is typical for a first-order transition. Only by artificially enlarging the number of components of the complex scalar field $\phi$ appearing in the Ginzburg-Landau model did Halperin, Lubensky, and Ma find an infrared stable fixed point, provided this number is taken to be larger as 183. However, the corresponding critical exponent $\eta$, which determines the anomalous dimension of $\phi$, depends on the gauge-fixing choice and is therefore unphysical. This should not come as a surprise since $\phi$ itself is not gauge invariant, and therefore not physical.

We shall carry out the renormalization group theory within the dual formulation which, being casted in terms of physical fields, does not suffer from the flaws of local gauge invariance [1]. The dual theory (74) involving a massive vector field is perturbatively renormalizable in four dimensions $(D=4)$ [35], so that usual perturbation theory can be applied to calculate the critical exponents. However, the derivation of the dual theory (74) from the Ginzburg-Landau model hinged on the fact that the number of space dimensions is three, for which the dual object $\epsilon_{i j k} \partial_{j} A_{k}$ is a vector. In other words, the dual theory describes the superconducting phase transition only in three dimensions. For this reason, we carry out the renormalization group theory in fixed $(D=3)$ dimension, and not in $D=4-\epsilon$ dimensions as is often done. The fixed-dimension approach to critical phenomena was introduced by Parisi, who applied it to a pure $|\psi|^{4}$ theory [36,14. The method makes explicitly use of the fact that near the critical point the system has only one relevant length scale, viz., the correlation length which diverges at this point. This length is used to convert dimensionful 
coupling constants into dimensionless ones.

In the present setting the relevant scale is the (renormalized) inverse mass $m_{\psi}^{-1}$. (The bare mass vanishes as $m_{\psi, 0}^{2} \sim T_{\mathrm{c}}-T$ at the critical temperature $T_{\mathrm{c}}$ ). We know from the GinzburgLandau theory that the bare penetration depth also diverges at $T_{\mathrm{c}}$, viz., $\lambda_{0} \sim\left(T_{\mathrm{c}}-T\right)^{-1 / 2}$. However, the renormalized length $\lambda$ should not constitute an independent diverging length scale. We will see below that this is indeed the case. As usual, the critical exponents are computed in the symmetric phase of the model, which in the present context corresponds to the superconducting phase.

We write the bare Hamiltonian (74) as a sum of the renormalized Hamiltonian and counterterms $\delta H$

$$
\begin{aligned}
\delta H=\int d^{3} x & {\left[\left(Z_{\psi}-1\right)|(\nabla-i g \mathrm{~h}) \psi|^{2}+\left(Z_{\psi} m_{\psi, 0}^{2}-m_{\psi}^{2}\right)|\psi|^{2}+u\left(Z_{u}-1\right)\left|\psi_{0}\right|^{4}\right.} \\
+ & \left.\frac{1}{2}\left(Z_{\mathrm{h}}-1\right)(\nabla \times \mathrm{h})^{2}+\frac{1}{2}\left(Z_{\mathrm{h}} m_{A, 0}^{2}-m_{A}^{2}\right) \mathrm{h}^{2}\right] .
\end{aligned}
$$

(All quantities appearing in (74) should have been given an index 0 to indicate that they refer to bare quantities. For convenience we rescaled the $\mathrm{h}$ field by a factor $m_{A}: \mathrm{h} \rightarrow m_{A} \mathrm{~h}$.) The renormalized objects are related to the bare ones via

$$
\mathrm{h}_{i}=Z_{\mathrm{h}}^{-1 / 2} \mathrm{~h}_{0, i}, \quad g=Z_{g}^{-1} Z_{\psi} Z_{\mathrm{h}}^{1 / 2} g_{0}, \quad \psi=Z_{\psi}^{-1 / 2} \psi_{0}, \quad u=Z_{u}^{-1} Z_{\psi}^{2} u_{0} .
$$

It is straightforward to calculate the one-loop diagrams. The correlation functions can be read off from the Hamiltonian (74). With a wiggly line denoting the correlation function of the vector field, and a straight line denoting the one of the $\psi$ field, it follows that

$$
\begin{aligned}
\sim \sim & \sim \frac{1}{\mathbf{k}^{2}+m_{A}^{2}}\left(\delta_{i j}-\frac{k_{i} k_{j}}{\mathbf{k}^{2}}\right) \\
& =\frac{1}{\mathbf{k}^{2}+m_{\psi}^{2}} .
\end{aligned}
$$

The correlation function (128a) reflects the constraint $\nabla \cdot \mathrm{h}=0$ which we imposed upon the fluctuating field $\mathrm{h}$. The correlation function has been obtained by representing the $\delta$ 
function $\delta(\nabla \cdot \mathrm{h})$ as $\exp \left[-\left(\nabla \cdot(\mathrm{h})^{2} /(2 \zeta)\right]\right.$ with $\zeta$ taken to zero at the end. We find for the diagrams depicted below

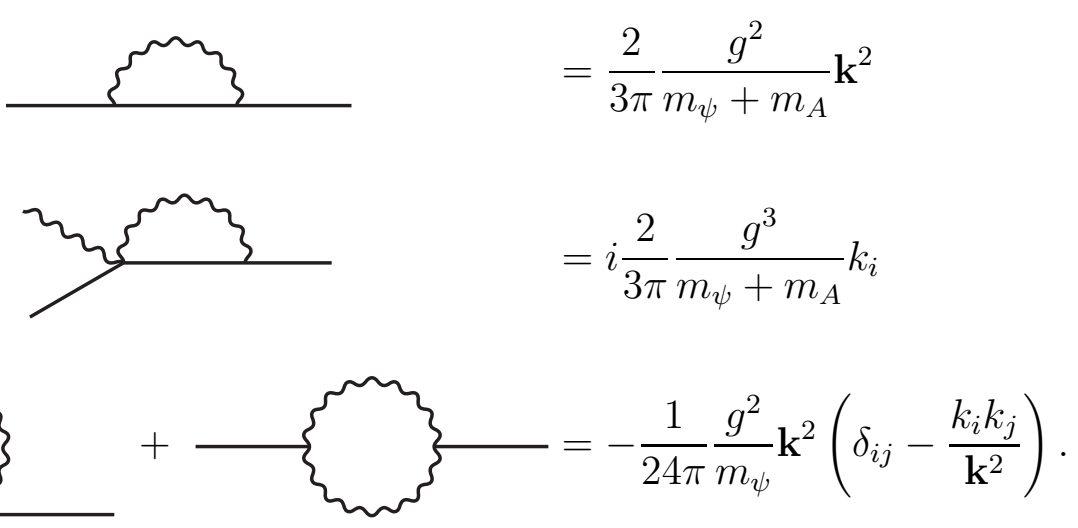

We considered these particular diagrams to illustrate the following observations. The first diagram yields a $Z_{\psi}$ factor given by

$$
Z_{\psi}=1+\frac{2}{3 \pi} \frac{g^{2}}{m_{\psi}+m_{A}} .
$$

From the second diagram we extract the factor $Z_{g}$,

$$
Z_{g}=1+\frac{2}{3 \pi} \frac{g^{2}}{m_{\psi}+m_{A}}
$$

which turns out to be equal to $Z_{\psi}$. To this order, the renormalized coupling constant $g$ is thus related to the bare one simply via

$$
g=Z_{\mathrm{h}}^{1 / 2} g_{0}
$$

This means that the minimal coupling to the vector field is preserved at the one-loop level. In fact, it is preserved to any order in the loop expansion. The reason is that the Ward identity which guarantees that the minimal coupling is always maintained in the case of a massless vector field, also operates in the massive case [35].

The diagrams (129d) show that the one-loop contributions to the self-energy of the vector field is transverse. This too remains true to all orders in perturbation theory thanks to the Ward identity. The mass term of the vector field is consequently not renormalized and does not need a counterterm. That is, $m_{A}=Z_{\mathrm{h}}^{1 / 2} m_{A, 0}$, so that the last term in $(\sqrt{126})$ vanishes. 
It follows that the critical exponent $\gamma_{\mathrm{h}}$ is unaffected by the fluctuations, and retains its mean-field value $\gamma_{\mathrm{h}}=1$.

Incidently, the electric charge does not renormalize in the dual theory since $g=(\pi / e) m_{A}$, and both $g$ and $m_{A}$ renormalize in the same manner.

We now come to an important observation related to the fact that in the dual theory the inverse penetration depth $m_{A}$ plays the role of a mass as well as of a coupling constant because $g=(\pi / e) m_{A}$, with $e$ a constant. The standard definition of the critical exponent $\nu$ which determines how the correlation length $m_{\psi}^{-1}$ diverges when the temperature approaches $T_{\mathrm{c}}: m_{\psi}^{-1} \sim\left(T_{\mathrm{c}}-T\right)^{-\nu}$, is

$$
\frac{1}{\nu}=\frac{\partial \ln \left(m_{\psi, 0}^{2}\right)}{\partial \ln \left(m_{\psi}\right)}
$$

In our case this can be rewritten as follows

$$
\frac{\partial m_{A, 0}^{2}}{\partial \ln \left(m_{\psi}\right)}=\frac{m_{A, 0}^{2}}{\nu}
$$

because $m_{\psi, 0}^{2} \sim m_{A, 0}^{2}$ near $T_{\mathrm{c}}$. The $\beta$ function is defined by the equation

$$
\beta\left(\hat{g}^{2}\right):=\left.m_{\psi} \frac{\partial}{\partial m_{\psi}} \frac{g^{2}}{m_{\psi}}\right|_{u_{0}, g_{0}},
$$

with the properly scaled coupling constant $\hat{g}^{2}:=g^{2} / m_{\psi}$. By virtue of (134), this can be cast in the form

$$
\beta\left(\hat{g}^{2}\right)=\hat{g}^{2}\left(-1+\frac{1}{\nu}+\gamma_{\mathbf{h}}\left(\hat{g}^{2}, \hat{u}\right) \frac{\partial \ln \left(m_{A}\right)}{\partial \ln \left(m_{\psi}\right)}\right),
$$

where $\gamma_{\mathbf{h}}\left(\hat{g}^{2}, \hat{u}\right)$, with $\hat{u}:=u / m_{\psi}$, is the function

$$
\gamma_{\mathrm{h}}\left(\hat{g}^{2}, \hat{u}\right):=\left.m_{A} \frac{\partial}{\partial m_{A}} \ln \left(Z_{\mathrm{h}}\right)\right|_{u_{0}, g_{0}}
$$

which yields the critical exponent $\eta_{\mathrm{h}}$ when evaluated at the critical point. Without the explicit mass dependence, the coefficient of the $\hat{g}^{2}$ term in the $\beta\left(\hat{g}^{2}\right)$ function would be -1 , implying that the origin $\hat{g}=0$ is an ultraviolet stable fixed point. In (136), however, the coefficient is $-1+1 / \nu$ which is positive if $\nu<1$. In this case, the origin $\hat{g}^{2}=0$ becomes 
infrared stable and the coupled theory reduces to a pure $|\psi|^{4}$ theory. The best estimate for $\nu$ available from summed perturbation theory at fixed $D=3$ [37 gives $\nu \approx .6695$, which is smaller than one. Hence, the trivial fixed point $\hat{g}^{*^{2}}=0$ is infrared stable (see Fig. 11). This situation differs dramatically from that in the Ginzburg-Landau theory where the coupling $e$ to the electromagnetic gauge field has an infrared stable fixed point away from the origin, see Fig. 12, and the corresponding value $\hat{e}^{*^{2}}$ is too large to allow the coupled system to develop an infrared stable fixed point.

To recapitulate, the dual theory of the superconducting phase transition possesses an infrared stable fixed point given by $\hat{g}^{*^{2}}=0$ and $\hat{u}^{*}=\hat{u}_{\mathrm{WF}}^{*}$, where $\hat{u}_{\mathrm{WF}}^{*}$ is the Wilson-Fisher fixed point of a pure $|\psi|^{4}$ theory with reversed temperature axis. The critical exponents of the $\psi$ field are the ones of a superfluid. The critical exponents pertaining to the $\mathrm{h}$ field, which physically represents the local induction, have their mean-field values. In particular, $\nu_{\mathrm{h}}=1 / 2$. This exponent reveals that the magnetic penetration depth diverges near $T_{\mathrm{c}}$ as $\left(T_{\mathrm{c}}-T\right)^{-1 / 2}$, meaning that inside the critical region the empirical formula $\lambda \sim[1-$ $\left.\left(T / T_{\mathrm{c}}\right)^{4}\right]^{-1 / 2}$ found outside this region remains unchanged.

A last point of interest is the Gaussian fixed point, corresponding to $\hat{g}^{*^{2}}=0, \hat{u}^{*}=0$. This fixed point is infrared stable in the $\hat{g}^{2}$ direction, and unstable in the $\hat{u}$ direction. It describes a loop gas of free vortices. At this point the phase transition changes from second to first order, i.e., it is a tricritical point, the existence and location of which was first established in Ref. [38]. At the level we are working, the critical exponents characterizing this point are Gaussian. A $|\psi|^{6}$ term which should now be included will generate logarithmic corrections.

\section{ACKNOWLEDGMENTS}

We thank A. Kovner and J. Rammer for useful discussions. 


\section{APPENDIX:}

Here we give a lattice version of the arguments presented in Sec. [II], showing the equivalence of a complex field theory and a loop gas [8,15]. We start with the free Hamiltonian (21) defined on a hypercubic lattice in $D$ space dimensions. (We use the same lattice notation as in Sec. [V). The corresponding energy expression we write as

$$
E=\sum_{\mathbf{x}, \mathbf{y}} a^{D-2} \psi^{*}(\mathbf{x}) \Lambda(\mathbf{x}, \mathbf{y}) \psi(\mathbf{y})
$$

where

$$
\Lambda(\mathbf{x}, \mathbf{y})=\left(2 D+m^{2} a^{2}\right) \delta_{\mathbf{x}, \mathbf{y}}-\sum_{i}\left(\delta_{\mathbf{x}, \mathbf{y}+a \mathbf{i}}+\delta_{\mathbf{x}, \mathbf{y}-a \mathbf{i}}\right)
$$

or in matrix notation

$$
\Lambda=\left(2 D+m^{2} a^{2}\right) I-S
$$

Here, $I$ is the identity operator and $S$ is the so-called step operator. The matrix element $S(\mathbf{x}, \mathbf{y})$ is 1 if the two lattice sites $\mathbf{x}$ and $\mathbf{y}$ are neighbors, and zero otherwise. From (A1) we immediately read off the lattice correlation function

$$
G(\mathbf{x})=a^{2-D} \Lambda^{-1}(0, \mathbf{x})
$$

The operator $\Lambda^{-1}$ we expand in a von Neumann series

$$
\Lambda^{-1}=\frac{\sigma}{2 D} \sum_{N=0}^{\infty}\left(\frac{S \sigma}{2 D}\right)^{N}
$$

where we introduced the abbreviation

$$
\sigma^{-1}=1+\frac{m^{2} a^{2}}{2 D}
$$

From the definition of the step operator $S$ it follows that $S^{N}(\mathbf{x}, \mathbf{y})$ is equal to the number of paths which go from site $\mathbf{x}$ to site $\mathbf{y}$ in $N$ steps. By virtue of this we can write

$$
\Lambda^{-1}(0, \mathbf{x})=\frac{\sigma}{2 D} \sum_{N=0}^{\infty} P_{N}(\mathbf{x}) \sigma^{N}
$$


with $P_{N}(\mathbf{x})$ the probability defined by the number of paths that go from 0 to $\mathbf{x}$ in $N$ steps divided by the ones that just start in 0 and contain $N$ steps. This last number is equal to $(2 D)^{N}$. The probability $P_{N}(\mathbf{x})$ plays a central role in the theory of random walks. A little thought reveals that it satisfies the recurrence relation

$$
P_{N+1}(\mathbf{x})=\frac{1}{2 D} \sum_{i} P_{N}(\mathbf{x}+a \mathbf{i})
$$

with the initial condition

$$
P_{0}(\mathbf{x})=\delta_{0, \mathbf{x}}
$$

To solve (A8) we introduce the Fourier transform:

$$
P_{N}(\mathbf{x})=a^{D} \int_{-\pi / a}^{\pi / a} \frac{d^{D} k}{(2 \pi)^{D}} e^{i \mathbf{k} \cdot \mathbf{x}} P_{N}(\mathbf{k}) .
$$

The recurrence relation then yields for $P_{N}(\mathbf{k})$

$$
P_{N+1}(\mathbf{k})=\frac{1}{D} \sum_{i} \cos \left(k_{i} a\right) P_{N}(\mathbf{k})
$$

with $P_{0}(\mathbf{k})=1$ as follows from the initial condition (A9). In this way we obtain as solution

$$
P_{N}(\mathbf{x})=a^{D} \int_{-\pi / a}^{\pi / a} \frac{d^{D} k}{(2 \pi)^{D}} \mathrm{e}^{i \mathbf{k} \cdot \mathbf{x}}\left[\frac{1}{D} \sum_{i} \cos \left(k_{i} a\right)\right]^{N} .
$$

We are now in a position to take the continuum limit $(a \rightarrow 0)$. Let us first concentrate on the probability (A12) and assume that the length $s$ of a path on a lattice is measured in some - as yet arbitrary - unit $b$. That is, a path of $N$ steps has length $s=N b$. Since in the limit $a \rightarrow 0$

$$
\left[\frac{1}{D} \sum_{i} \cos \left(k_{i} a\right)\right]^{N} \rightarrow\left(1-\frac{a^{2}}{2 D} \mathbf{k}^{2}\right)^{s / b} \rightarrow \exp \left(-s \frac{a^{2}}{2 D b} \mathbf{k}^{2}\right)
$$

we have to take the ratio $a^{2} / b$ fixed in order to obtain a non-trivial continuum limit; we will take $a^{2} / b=2 D$. In other words, $b \sim a^{2}$ in the scaling limit. In this limit the probability density $p(\mathbf{x}, s):=\lim _{a \rightarrow 0} P_{N}(\mathbf{x}) / a^{D}$ becomes

$$
p(\mathbf{x}, s)=\int \frac{d^{D} k}{(2 \pi)^{D}} \mathrm{e}^{i \mathbf{k} \cdot \mathbf{x}} \mathrm{e}^{-s \mathbf{k}^{2}},
$$


so that the correlation function (A4), with $\Lambda^{-1}$ given by (A5), can be written as

$$
\begin{aligned}
G(\mathbf{x}) & =\lim _{a \rightarrow 0} a^{2-D} \Lambda^{-1}(0, \mathbf{x})=\lim _{a \rightarrow 0} \frac{a^{2} \sigma}{2 D} \sum_{N=0}^{\infty} \frac{1}{a^{D}} P_{N}(\mathbf{x})\left(1+\frac{m^{2} a^{2}}{2 D}\right)^{-s / b} \\
& =\int_{0}^{\infty} \mathrm{e}^{-s m^{2}} p(\mathbf{x}, s),
\end{aligned}
$$

where we used the fact that $\sigma \rightarrow 1$ and

$$
\frac{a^{2}}{2 D} \sum_{N=0}^{\infty} \rightarrow \int_{0}^{\infty} d s
$$

when $a \rightarrow 0$. But with $p(\mathbf{x}, s)$ given in (A14), this is precisely the Schwinger representation (23) of the correlation function.

A similar derivation can be given for the partition function. To this end we consider the probability $P_{N}^{\mathrm{c}}$ defined by the number of closed oriented paths containing $N$ steps divided by $(2 D)^{N}$. It is related to the probability $P_{N}(\mathbf{x})$ previously introduced via

$$
P_{N}(0)=N P_{N}^{\mathrm{c}}
$$

where the factor $\mathrm{N}$ arises from the fact that one can start traversing a given oriented loop at any of the $N$ lattice sites visited by the loop. It then easily follows that

$$
\sum_{N=0}^{\infty} \frac{P_{N}^{\mathrm{c}}}{a^{D}} \sigma^{N} \rightarrow \int_{0}^{\infty} \frac{d s}{s} \mathrm{e}^{-s m^{2}} \int \frac{d^{D} k}{(2 \pi)^{D}} \mathrm{e}^{-s \mathbf{k}^{2}}
$$

which is the right-hand side of the first equation in (27). This leads to the same representation of the partition function we found before with help of Schwinger's proper-time representation. The steric repulsion can also be included on the lattice, but this will not be done here. 


\section{REFERENCES}

[1] M. Kiometzis, H. Kleinert, and A. M. J. Schakel, Phys. Rev. Lett. 73, 1975 (1994).

[2] T. Banks, B. Meyerson, and J. Kogut, Nucl. Phys. B 129, 493 (1977).

[3] M. Peskin, Ann. Phys. 113, 122 (1978).

[4] P. R. Thomas and M. Stone, Nucl. Phys. B 144, 513 (1978).

[5] R. Savit, Rev. Mod. Phys. 52, 453 (1989).

[6] V. L. Berezinskii, Zh. Eksp. Teor. Fiz. 61, 1144 (1971) [Sov. Phys. JETP 34, 610 (1972)].

[7] J. M. Kosterlitz and D. J. Thouless, J. Phys. C 6, 1181 (1973).

[8] H. Kleinert, Gauge Fields in Condensed Matter (World Scientific, Singapore, 1989) Vol. 1.

[9] A. Kovner, B. Rosenstein, and D. Eliezer, Mod. Phys. Lett A 5, 2733 (1990); Nucl Phys. B 350, 325 (1991); A. Kovner and B. Rosenstein, Phys. Rev. Lett. 67, 1490 (1991).

[10] S. Elitzur, Phys. Rev. D 12, 3978 (1975).

[11] R. P. Feynman, Rev. Mod. Phys. 20, 367 (1948).

[12] J. Schwinger, Phys. Rev. 128, 2425 (1962).

[13] F. A. Schaposnik, Phys. Rev. D 18, 1183 (1978).

[14] G. Parisi, Statistical Field Theory (Addison-Wesley, New-York, 1988).

[15] J. Ambjørn, Fortschr. Phys. 36, 595 (1988); C. Itzykson and J. -M. Drouffe, Statistical Field Theory (Cambridge Univ. Press, Cambridge, 1989).

[16] J. Schwinger, Phys. Rev. 82, 664 (1951).

[17] J. Villain, J. Physique 36, 591 (1975).

[18] A. Kovner, P. Kurzepa, and B. Rosenstein, Mod. Phys. Lett. A 8, 1343 (1993). 
[19] M. Kiometzis and A. M. J. Schakel, Int. J. Mod. Phys. B 7, 4271 (1993).

[20] P. A. M. Dirac, Phys. Rev. 74, 817 (1948).

[21] H. Kleinert, Int. J. Mod. Phys. 7, 4693 (1992).

[22] Y. Nambu, Phys. Rev. D 10, 4262 (1974).

[23] A. A. Abrikosov, Sov. Phys. JETP 5, 1174 (1957).

[24] E. C. Marino, Phys. Rev. D 38, 3194 (1988); see also E. C. Marino, G. C. Marques, R. O. Ramos, and J. S. Ruiz, Phys. Rev. D 45, 3690 (1992); E. C. Marino, Ann. Phys. (NY) 224, 225 (1993).

[25] K. Bardakci and S. Samuel, Phys. Rev. D 18, 2849 (1978).

[26] H. Kawai, Prog. Theor. Phys. 65, 351 (1981).

[27] D. Saint-James, E. J. Thomas, and G. Sarma, type-II Superconductivity (Pergamon, Oxford, 1969).

[28] L. Kramer, Z. Phys. 258, 367 (1973); M. C. Leung and A. E. Jacobs, J. Low Temp. Phys. 11, 395 (1973).

[29] P. G. de Gennes, Superconductivity of Metals and Alloys (Addison-Wesley, New York, 1966).

[30] J. Rammer, Ph. D. Thesis, Universität Bayreuth, 1988.

[31] A. L. Fetter, in The Physics of Liquid and Solid He, Part I, edited by K. H. Bennemann and J. B. Ketterson (J. Wiley, New York, 1976).

[32] B. I. Halperin, T. C. Lubensky and S. Ma, Phys. Rev. Lett. 32, 292 (1974).

[33] H. Kleinert and A. M. J. Schakel, One-loop critical exponents for Ginzburg-Landau theory with Chern-Simons term (unpublished). 
[34] S. Coleman and E. Weinberg, Phys. Rev. D 7, 1888 (1973).

[35] J. C. Collins, Renormalization (Cambridge Univ. Press, Cambridge, 1984).

[36] G. Parisi, Lectures presented at the Cargèse Summer School 1973 (unpublished); J. Stat. Phys. 23, 49 (1980).

[37] J. Zinn-Justin, Quantum Field Theory and Critical Phenomena (Oxford Univ. Press, Oxford, 1989).

[38] H. Kleinert, Lett. Nuovo Cimento 35, 405 (1982). 


\section{FIGURES}

FIG. 1. Magnetic field (thin lines) of a positively charged monopole. The magnetic flux is provided by the Dirac string (thick line) located along the negative $z$ axis.

FIG. 2. Biot-Savart interaction (wiggly line) between two line elements $d x_{i}$ and $d y_{i}$ of a magnetic vortex (straight line).

FIG. 3. Triangular Abrikosov flux lattice. The two vectors $\mathbf{v}_{1}$ and $\mathbf{v}_{2}$ span the unit cell, containing one flux tube.

FIG. 4. Magnetic induction $B$ versus applied magnetic field $H$ in arbitrary units for a type-II superconductor.

FIG. 5. Schematic representation of the interaction potential $V(r)$ between two parallel magnetic vortices for a type-II/1 superconductor.

FIG. 6. Contour plots of the local induction $h$ in an Abrikosov flux lattice for a type-II superconductor with $\kappa=1.3$. The first plot is at $B / H_{\mathrm{c}_{2}}=0.1$ and the second is at $B / H_{\mathrm{c}_{2}}=0.9$ (after Ref. [30]).

FIG. 7. A magnetic monopole with its string piercing the surface $\Sigma$ at $\partial \Sigma$.

FIG. 8. Two magnetic moments $\mathbf{m}_{I}$ and $\mathbf{m}_{I I}$ interacting with each other. The first one is kept fixed, while the second is free to rotate.

FIG. 9. Solid angle $\Omega$ that the vortex loop $\mathbf{J}$ subtends at the point $\mathbf{x}$.

FIG. 10. Two different surfaces spanning the loop $L$. The flux through the surfaces differ by a factor $4 \pi$.

FIG. 11. The $\beta$ function for the dual coupling constant (squared) $\hat{g}^{2}=g^{2} / m_{\psi}$, showing that the origin is an infrared stable fixed point. 
FIG. 12. The $\beta$ function for the electric charge (squared) $\hat{e}=g / m_{\psi}$ as obtained from the Ginzburg-Landau theory, showing that the origin is an infrared unstable fixed point. 


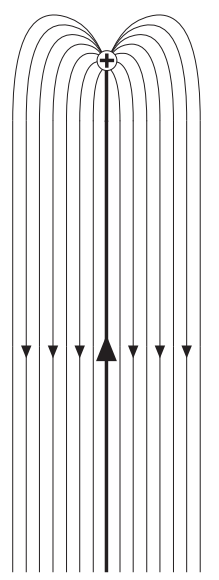

FIG. 1

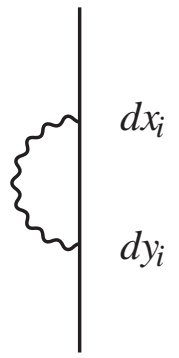

FIG. 2 


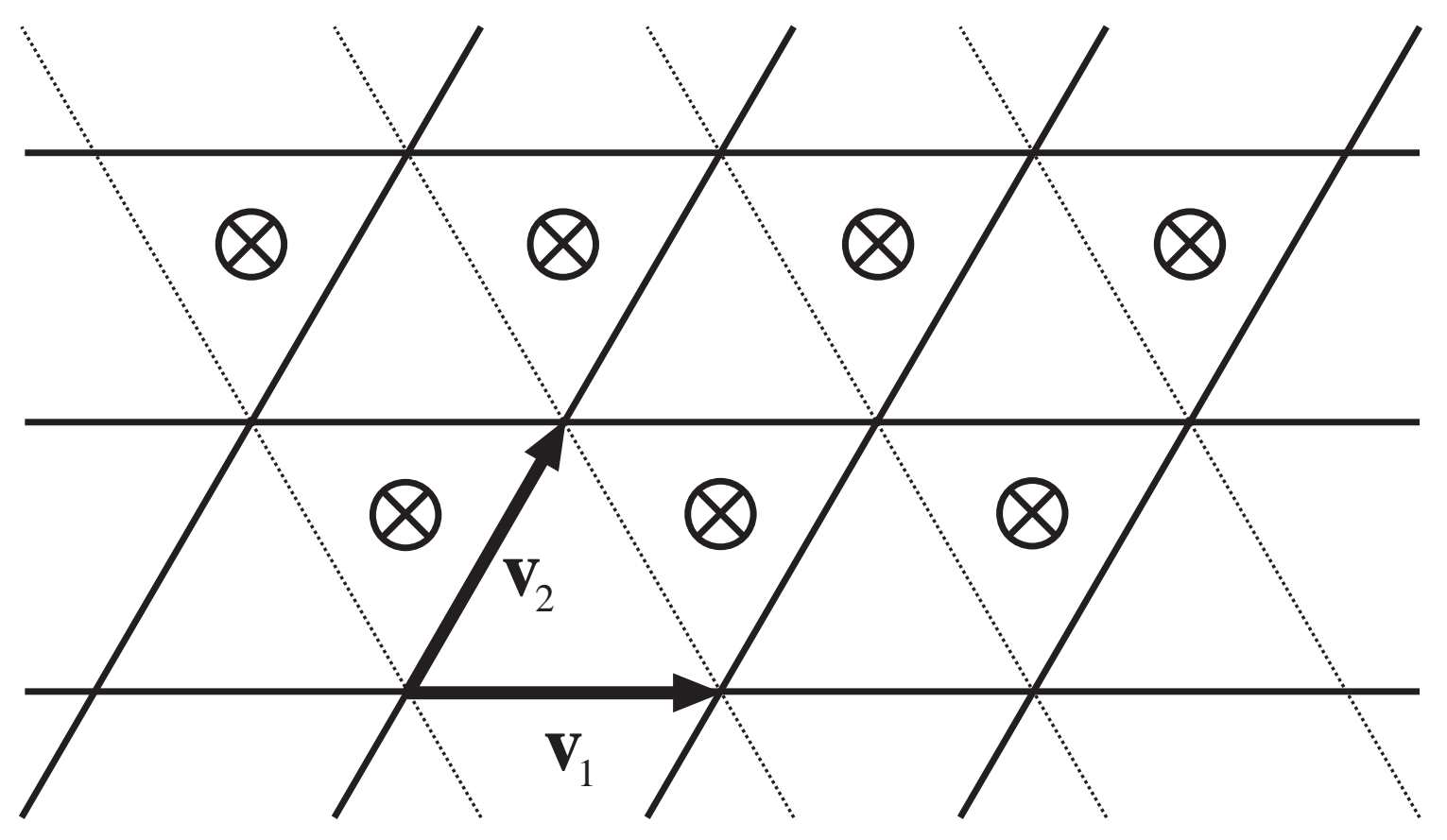

FIG. 3

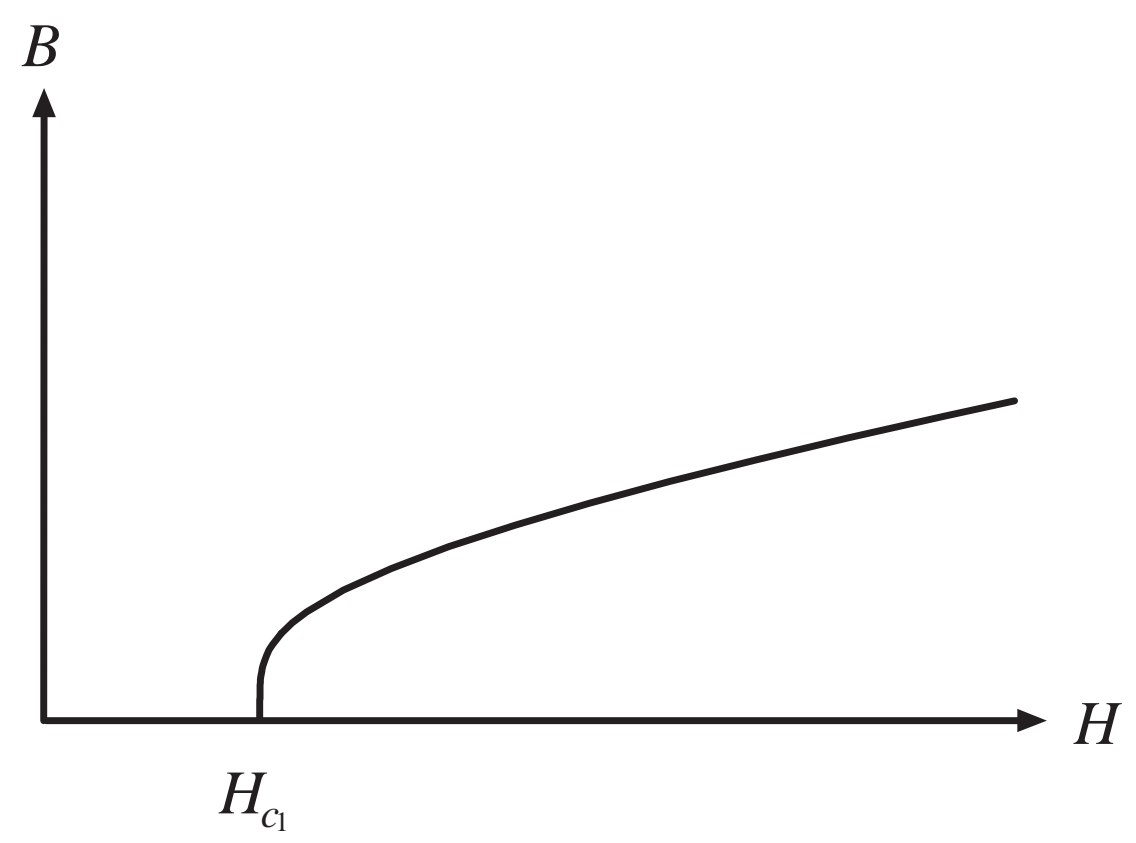

FIG. 4 


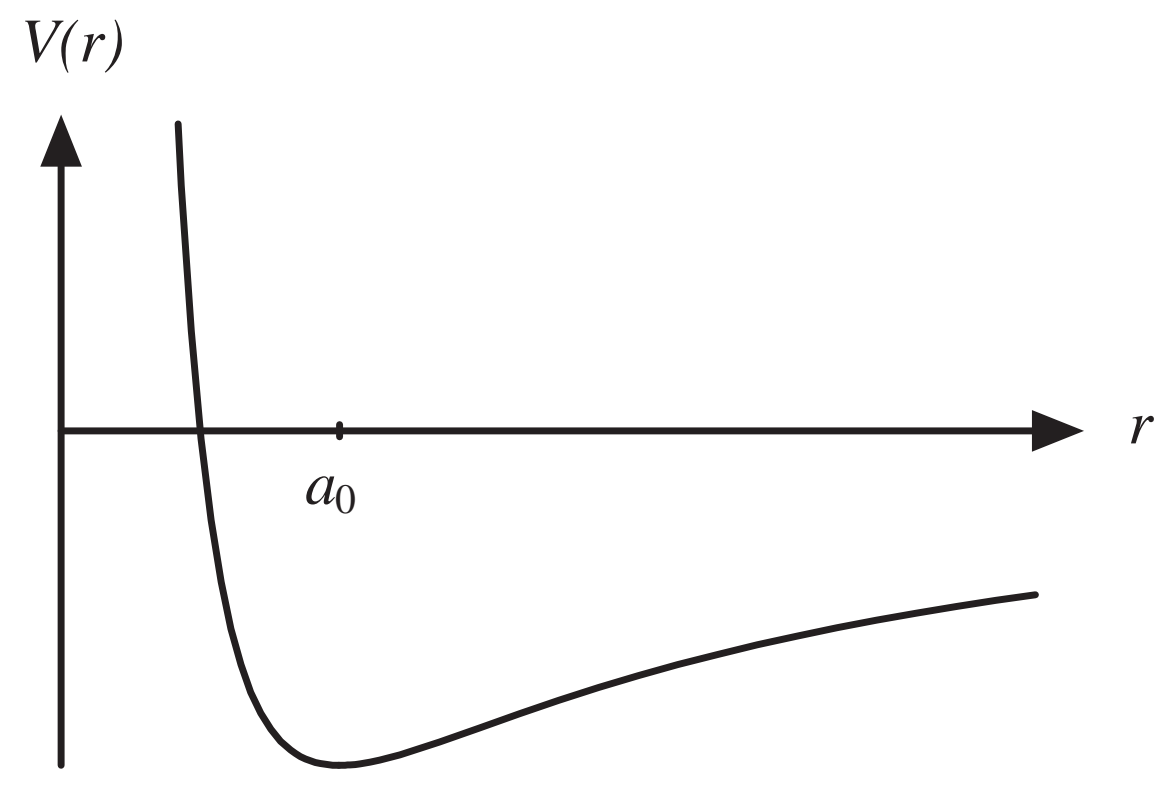

FIG. 5
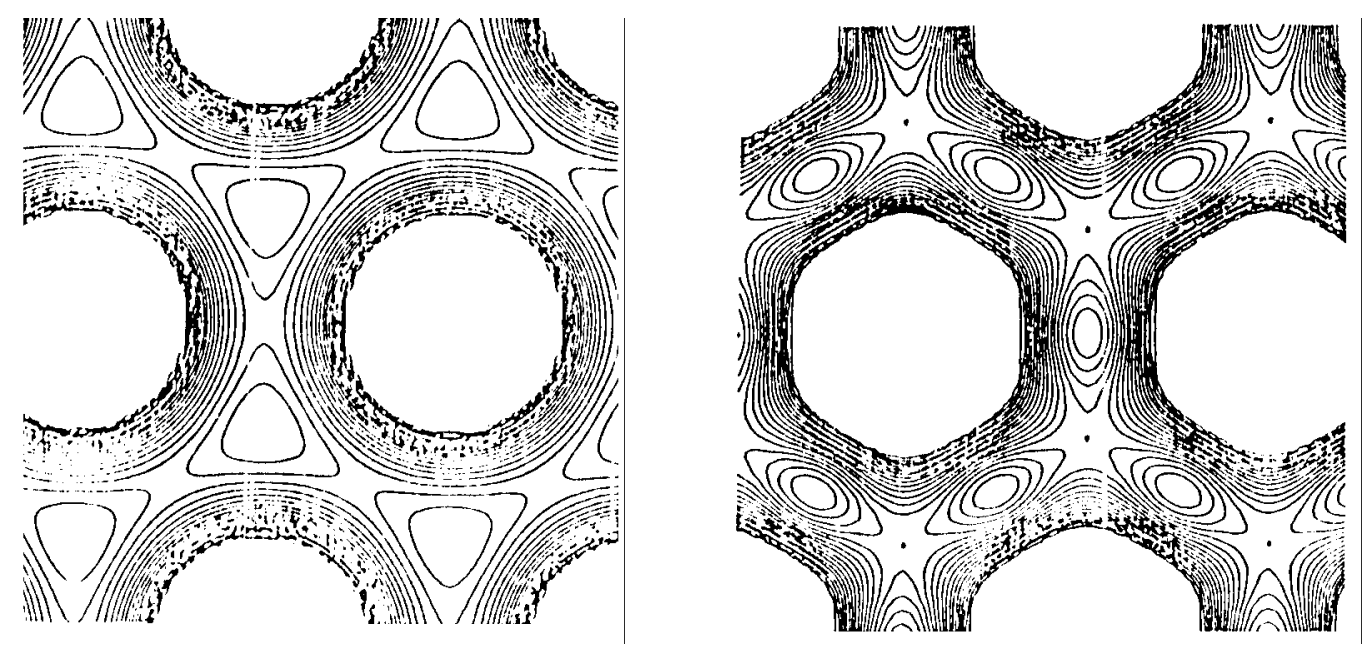

FIG. 6 


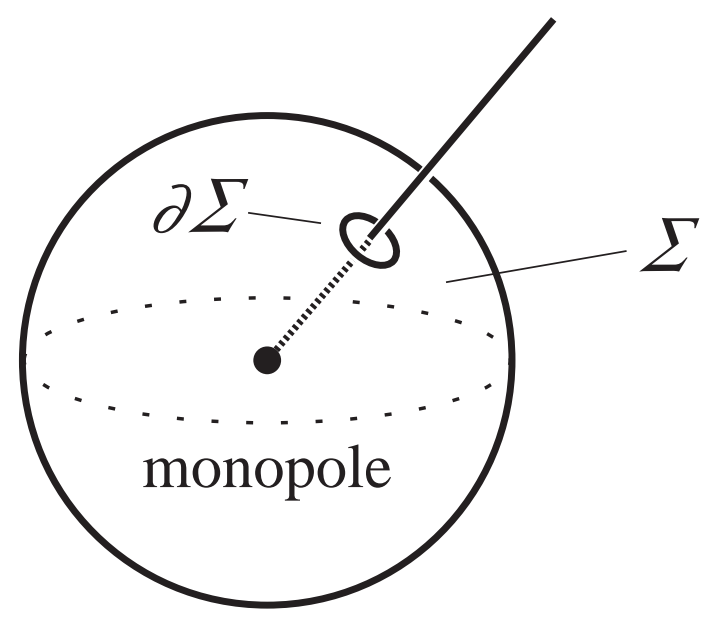

FIG. 7
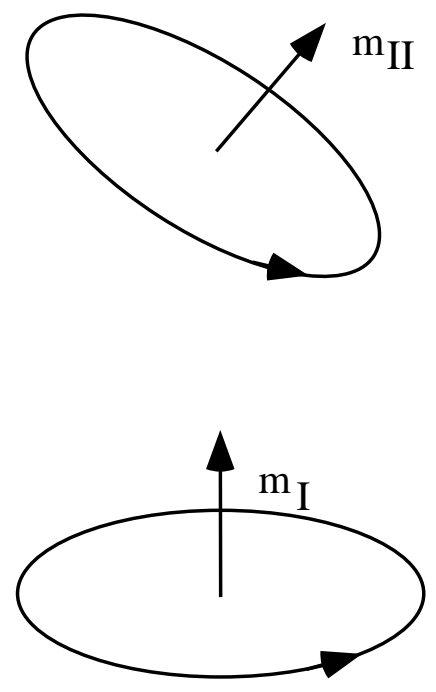

FIG. 8 


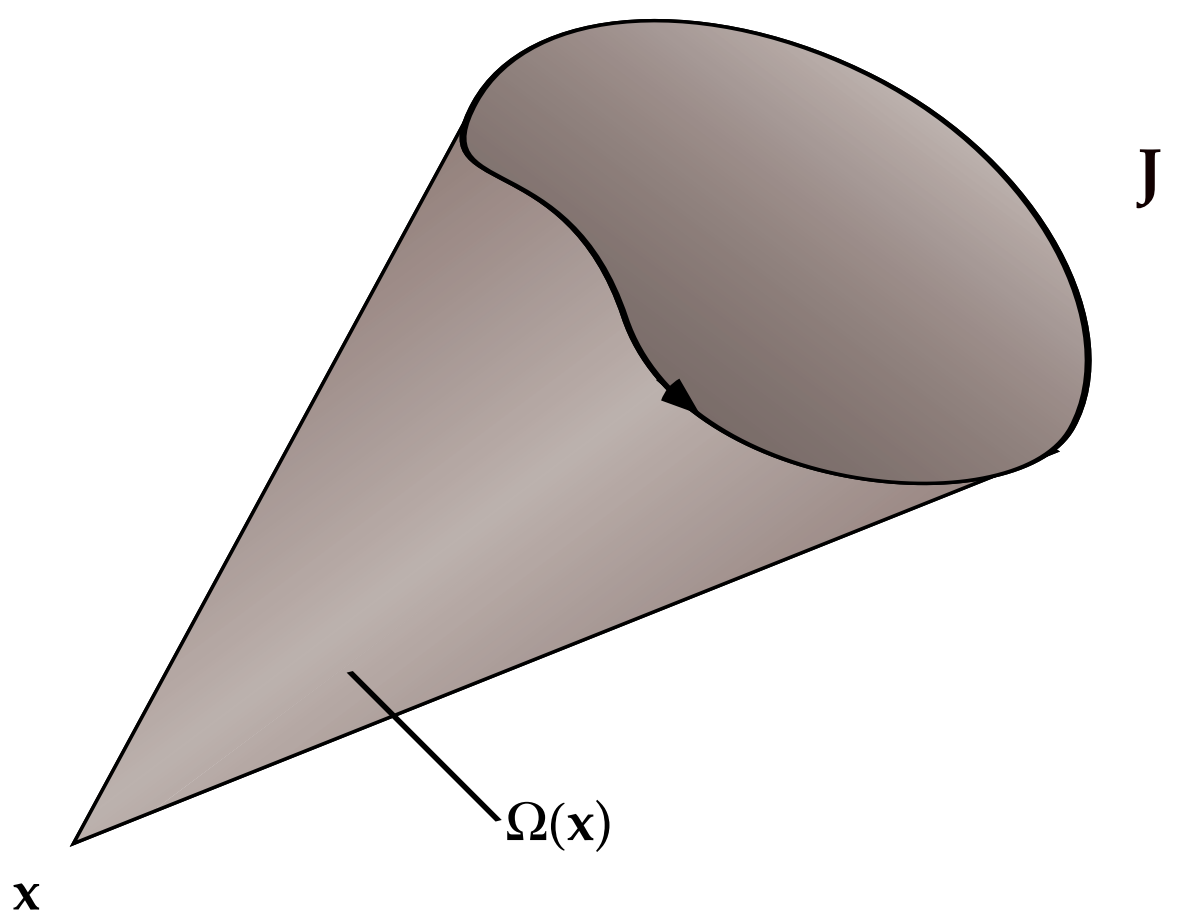

FIG. 9 


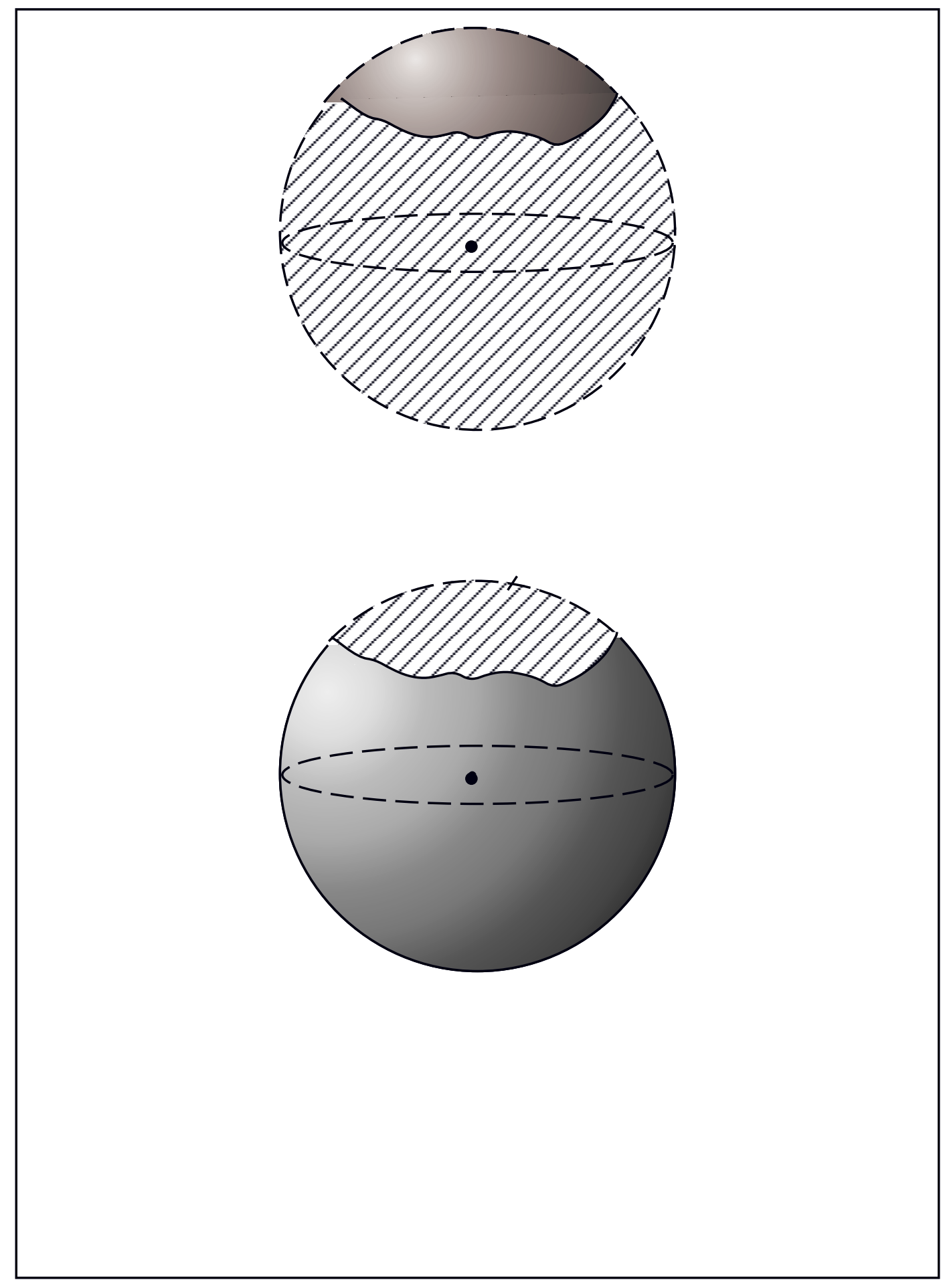

FIG. 10 


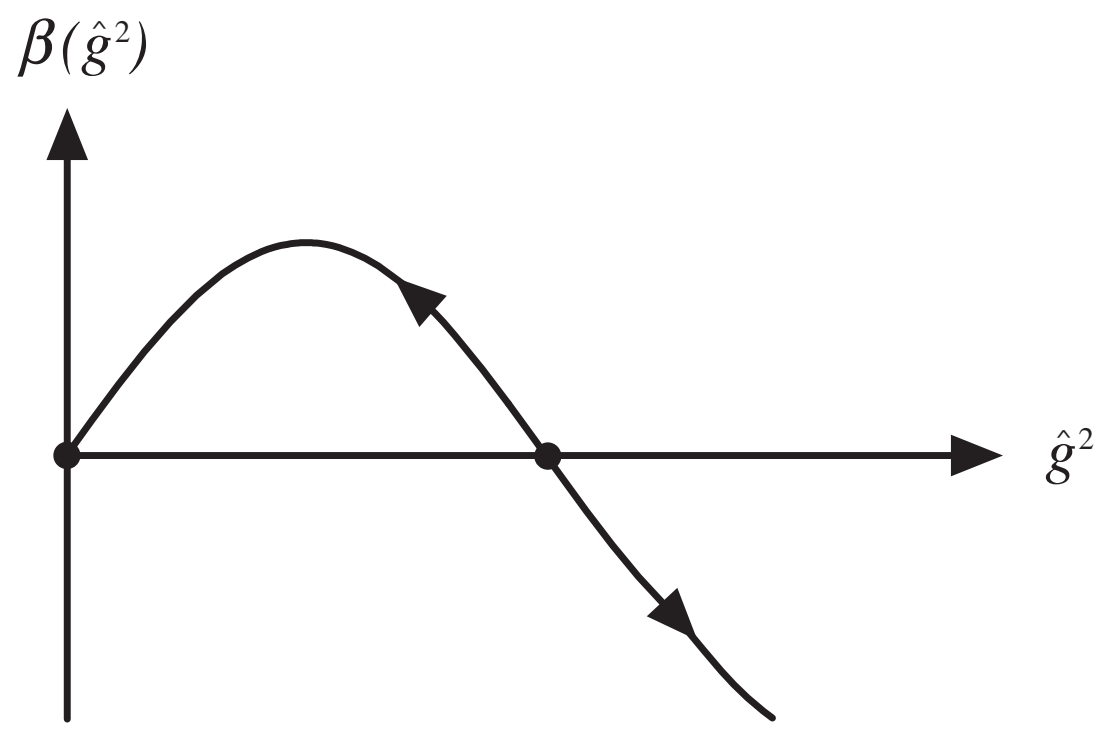

FIG. 11

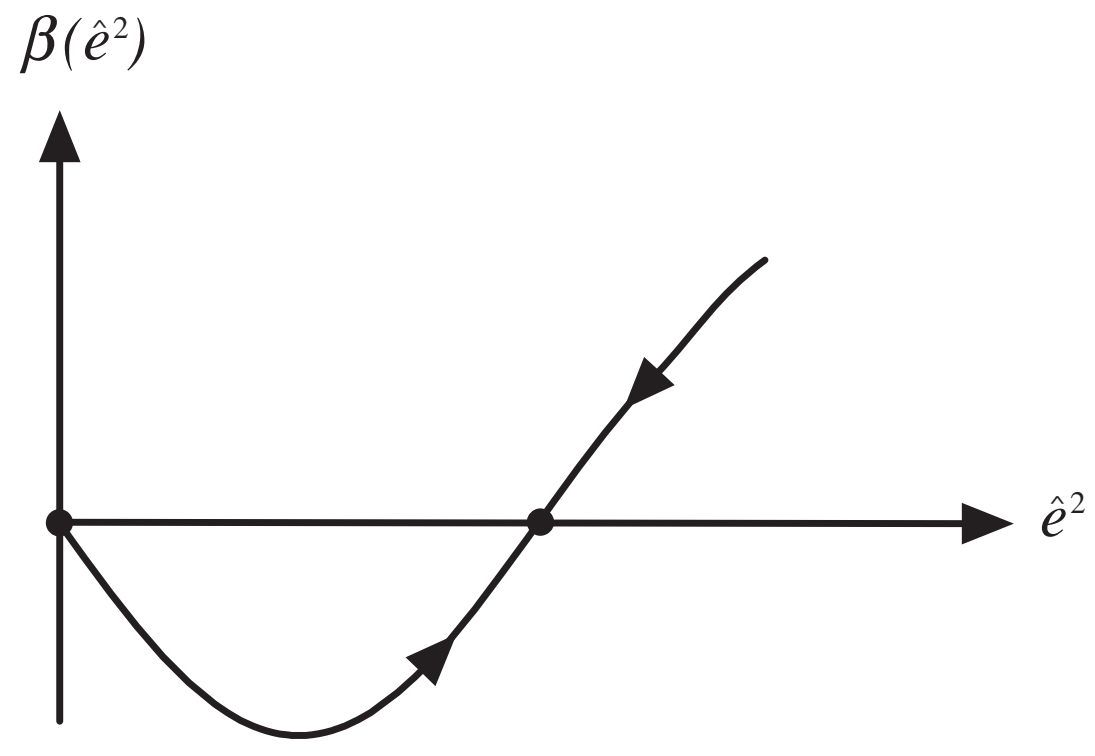

FIG. 12 